\title{
Glyphosate inhibits melanization and increases susceptibility to infection in insects
}

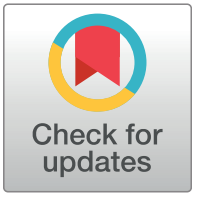

\section{G openaccess}

Citation: Smith DFQ, Camacho E, Thakur R, Barron AJ, Dong Y, Dimopoulos G, et al. (2021) Glyphosate inhibits melanization and increases susceptibility to infection in insects. PLoS Biol 19(5): e3001182. https://doi.org/10.1371/journal. pbio.3001182

Academic Editor: Anita Sil, University of California, San Francisco, UNITED STATES

Received: June 8, 2020

Accepted: March 11, 2021

Published: May 12, 2021

Peer Review History: PLOS recognizes the benefits of transparency in the peer review process; therefore, we enable the publication of all of the content of peer review and author responses alongside final, published articles. The editorial history of this article is available here: https://doi.org/10.1371/journal.pbio.3001182

Copyright: @ 2021 Smith et al. This is an open access article distributed under the terms of the Creative Commons Attribution License, which permits unrestricted use, distribution, and reproduction in any medium, provided the original author and source are credited.

Data Availability Statement: The datasets presented in this manuscript are publicly available Figshare data repository at: https://figshare.com/ projects/Glyphosate_Inhibits_Melanization_and_

\author{
Daniel F. Q. Smith ${ }^{1}$, Emma Camacho ${ }^{1}$, Raviraj Thakur ${ }^{20 ম}$, Alexander J. Barron ${ }^{30}$, \\ Yuemei Dong ${ }^{1}$, George Dimopoulos ${ }^{1}$, Nichole A. Broderick $\oplus^{3 \neq}$, Arturo Casadevall $\oplus^{1 \neq \mathrm{a}_{*}}$ \\ 1 W. Harry Feinstone Department of Molecular Microbiology and Immunology, Johns Hopkins Bloomberg \\ School of Public Health, Baltimore, Maryland, United States of America, 2 Department of Otolaryngology, \\ Head and Neck Surgery, Johns Hopkins Medicine, Baltimore, Maryland, United States of America, \\ 3 Department of Biology, Johns Hopkins University, Baltimore Maryland, United States of America \\ (- These authors contributed equally to this work. \\ a Current address: Cancer Early Detection Advanced Research Center, Knight Cancer Institute, Oregon \\ Health and Science University, Portland, Oregon, United States of America \\ $\ddagger \mathrm{NAB}$ and $\mathrm{AC}$ are joint senior authors on this work. \\ * acasade1@jhu.edu
}

\section{Abstract}

Melanin, a black-brown pigment found throughout all kingdoms of life, has diverse biological functions including UV protection, thermoregulation, oxidant scavenging, arthropod immunity, and microbial virulence. Given melanin's broad roles in the biosphere, particularly in insect immune defenses, it is important to understand how exposure to ubiquitous environmental contaminants affects melanization. Glyphosate-the most widely used herbicide globally_inhibits melanin production, which could have wide-ranging implications in the health of many organisms, including insects. Here, we demonstrate that glyphosate has deleterious effects on insect health in 2 evolutionary distant species, Galleria mellonella (Lepidoptera: Pyralidae) and Anopheles gambiae (Diptera: Culicidae), suggesting a broad effect in insects. Glyphosate reduced survival of $G$. mellonella caterpillars following infection with the fungus Cryptococcus neoformans and decreased the size of melanized nodules formed in hemolymph, which normally help eliminate infection. Glyphosate also increased the burden of the malaria-causing parasite Plasmodium falciparum in A. gambiae mosquitoes, altered uninfected mosquito survival, and perturbed the microbial composition of adult mosquito midguts. Our results show that glyphosate's mechanism of melanin inhibition involves antioxidant synergy and disruption of the reaction oxidation-reduction balance. Overall, these findings suggest that glyphosate's environmental accumulation could render insects more susceptible to microbial pathogens due to melanin inhibition, immune impairment, and perturbations in microbiota composition, potentially contributing to declines in insect populations.

\section{Introduction}

Melanin, a black-brown pigment found in all biological kingdoms, is produced through a series of oxidation and reduction reactions. These reactions are typically catalyzed by 2 classes 
Increases_Susceptibility_to_Infection_in_Insects/ 99341. Additionally, the Figshare Digital Object Identifiers (DOIs) for the individual figure datasets are listed in S1 Table.

Funding: D.F.Q.S., E.C., A.C. are funded by National Institute of Allergy and Infection Disease R01 Al052733 and Johns Hopkins Malaria Research Institute (https://malaria.jhsph.edu/) Pilot Grant \# Casadevall_123. D.F.Q.S. is funded by National Institutes of Health 5T32GM008752-18 and 1T32Al138953-01A1. A.J.B. and N.A.B. are funded by National Institutes of Health R35GM128871. The funders had no role in study design, data collection and analysis, decision to publish, or preparation of the manuscript. The salaries of D.F. Q.S., A.C., and E.C. are in part funded by the National Institute of Allergy and Infection Disease. The salaries of D.F.Q.S., A.J.B., and N.A.B., are in part funded by the National Institutes of Health. The salary of E.C. is in part funded by Johns Hopkins Malaria Research Institute.

Competing interests: The authors have declared that no competing interests exist.

Abbreviations: ABTS, 2,2'-azino-bis(3ethylbenzothiazoline-6-sulfonic acid); AMPA, aminomethylphosphonic acid; CFU, colony forming unit; DPI, days postinfection; L-DOPA, 3,4dihydroxyphenylalanine; OTU, operational taxonomic unit; $\mathrm{PCOA}$, principal coordinates analysis; ROS, reactive oxygen species. of enzymes: laccases (EC. 1.10.3.2) and phenoloxidases-a family which includes tyrosinases (EC. 1.14.18.1) [1]. Tyrosinases are copper metalloenzymes found in bacteria, fungi, protists, arthropods, birds, and mammals [2-7], and have 2 catalytic roles: (1) hydroxylation of monophenols into ortho-diphenols, followed by (2) oxidation of $o$-catechols into $o$-quinones [8]. During melanization, tyrosinase converts 3,4-dihydroxyphenylalanine (L-DOPA) to dopaquinone. Dopaquinone undergoes oxidation and reduction reactions to first form dopachrome, then dihydroxyindole. Dihydroxyindole undergoes radical-mediated polymerization to form melanins [8,9].

Melanization is an important component of immunity in virtually all insects [9]. Upon infection, protease cascades are activated that cleave pro-phenoloxidases into active phenoloxidases. Phenoloxidases convert catecholamines in the hemolymph into melanin, which surrounds and eliminates the pathogen through exposure to reactive oxygen species (ROS) and lysis from toxic melanin intermediates [3,9-13]. This melanization process is a key component of insect immune defense against bacterial, fungal, and protozoan pathogens, nematode parasites, and insect parasitoids [14-21]. In addition, pathogens are cleared by 2 similar processes: nodulation of smaller microbes such as bacteria, fungi, and protozoa, and encapsulation for infections with larger organisms such as helminths and parasitoid eggs [22]. Both nodulation and encapsulation involve pathogen neutralization via melanin accumulation and hemocyte (insect "blood cells") aggregation around the pathogen [22]. Melanization and phenoloxidases are also important for wound healing and cuticular development-processes vital for insect health and survival $[23,24]$. Since melanization is an essential physiological process and effector of insect health, understanding how common environmental contaminants affect melanin production is important. The significance of this is also highlighted by findings suggesting that insect populations may be in decline in recent decades [25].

One ubiquitous chemical found in the environment is glyphosate, the most commonly used herbicide worldwide, which was previously shown to interfere with melanization in the fungus Cryptococcus neoformans [26]. Glyphosate is a phosphonic glycine analogue and the active ingredient in Roundup herbicides [27]. It kills plants through competitive inhibition of EPSP synthase in the shikimate pathway responsible for aromatic amino acid synthesis in many plants, fungi, and bacteria [28]. The low cost of glyphosate and wide availability of genetically modified glyphosate-resistant crops has increased both crop yields and glyphosate-based herbicide use in agriculture [29,30]. From 1996 to 2014, glyphosate-resistant crops were linked to a 12-fold global increase in glyphosate use, including 8-fold in the United States, 134-fold in Brazil, and 107-fold in Argentina [31,32].

In practice, glyphosate is commonly applied at concentrations of approximately 28 to 57 $\mathrm{mM}$ [33] or in formulations of $360 \mathrm{~g} / \mathrm{L}$ (2 M), with $720 \mathrm{~g}$ (4 mol) per hectare [34]. Glyphosatebased herbicides are sprayed onto crops where the glyphosate is taken up by plant leaves and translocated to growing tissues throughout the plant [35]. Glyphosate is translocated to the roots where it is released into the soil [34]. In total, about $88 \%$ of the sprayed glyphosate ends up in the topsoil [36-38]. Less than 1\% of glyphosate has been shown to enter water bodies, typically following heavy rain, snowmelt, ploughing, or erosion [37], but concentrations from $<1 \mathrm{nM}$ to approximately $30 \mu \mathrm{M}$ in nearby water have been reported [39]. Further, glyphosate has been shown to enter the air through wind erosion and deposit via rain [40].

Glyphosate is remarkably stable, with half-life ranging from weeks to years depending on the surrounding microbial populations, which provide the primary mechanism of glyphosate degradation, while temperature, light, acidity, and salinity also play roles in the degradation process. Microbes mostly break down glyphosate into aminomethylphosphonic acid (AMPA), which persists up to 20 times longer than glyphosate and is often found in higher concentrations in topsoil and water [41-45]. 
While glyphosate may have harmful effects on microbes and animals (as reviewed in $[35,46])$, its impact on environmental microbial communities is inconclusive. Some studies demonstrate clear perturbation of microbial communities, including disrupting rhizosphere composition and fungal endophyte growth and viability [47-50], while others show little to no long-term impact on microbial communities [51-53], with no effects on overall soil health or reduction in soil microbial mass [54]. Microbial communities are also abundant in insect guts, where they are important for insect health [55-58], and several studies have linked detrimental effects of glyphosate on insect health to disruption of the microbiota. Honeybees exposed to glyphosate have altered microbiomes and are more susceptible to Serratia marcescens [59], although AMPA did not have the same effect [60]. In tsetse fly midguts, glyphosate disrupts Wigglesworthia glossinidia's production of folate-a compound required for tsetse fly health and vector competence for Trypanosoma brucei parasites [61].

Beyond effects on microbial communities, glyphosate has broad physiological impacts on insects, other arthropods, and vertebrates. While glyphosate was harmless to Lepthyphantes tenuis spiders, it changed behavior and increased mortality in Pardosa milvina and Neoscona theisi spiders [62-64]. Glyphosate reduced learning in Aedes aegypti mosquitoes [65] and in honeybees reduced survival and caused learning defects associated with feeding, homing, and flight behaviors [66,67]. Glyphosate and AMPA delayed development and reduced survival of the arthropod Daphnia magna [68]. Glyphosate induces oxidative stress and damage in many organisms, including D. magna, insects (fruit flies), amphibians (African clawed frog, European green toad, marsh frog), fish (brown trout, spotted snakehead fish), and mammals (rats) [69-74], often linked with lipid peroxidation and expression of antioxidant defenses (catalase, glutathione, and superoxide dismutase). In human erythrocytes, glyphosate and AMPA mixtures increase ROS production [75,76].

Given the melanin-inhibitory properties of glyphosate in fungi [26], we examined the roles of glyphosate and AMPA as inhibitors of insect melanization and phenoloxidase. We used 2 distinct insect models that both rely on melanin-based immunity: Galleria mellonella, a species of wax moths (Lepidoptera: Pyralidae), and Anopheles gambiae, a mosquito vector of malaria (Diptera: Culicidae). Considering melanin's importance in insect immunity, we evaluated glyphosate's effects on G. mellonella susceptibility to the pathogenic fungus C. neoformans, and on A. gambiae survival and susceptibility to the malaria parasite Plasmodium falciparum as measured by parasite oocyst burden. Additionally, we evaluated glyphosate's mechanism of melanin inhibition using L-DOPA auto-oxidation and mushroom tyrosinase-mediated oxidation models. Mushroom tyrosinase is commercially available and produces melanin in a similar mechanism as insect phenoloxidase. The purified enzyme and L-DOPA auto-oxidation allowed us to take a controlled step-by-step biochemical approach to show that glyphosate inhibits melanization by disrupting oxidative balance.

\section{Results}

\section{Glyphosate and AMPA inhibit Galleria mellonella phenoloxidase activity}

In insects, phenoloxidases are activated by serine proteases upon wounding or infection, thus triggering melanin production to either clot a wound or restrict a pathogen [10]. To investigate whether glyphosate inhibited insect melanogenesis, we used 2 models: G. mellonella wax moth larvae, and A. gambiae adult mosquitoes, a main vector of malaria.

In an ex vivo analysis using G. mellonella hemolymph, we found that glyphosate inhibited phenoloxidase activity in a dose-dependent manner, without addition of exogenous substrate (Fig 1A). Similar results were found with addition of a broad-spectrum protease inhibitor, which was used to control for continued activation of phenoloxidase, glyphosate-induced 
cellular responses, and/or off-target effects on other components of the phenoloxidase cascade (S1A Fig). We also saw similar inhibition of phenoloxidase activity with the addition of exogenous L-DOPA; however, in these experiments, there was only a modest enhancement of phenoloxidase activity with lower glyphosate concentrations followed by striking inhibition at higher concentrations (S1B Fig). Importantly, glyphosate did not impact hemocyte viability, as measured by trypan blue exclusion (S1C Fig). AMPA, a primary breakdown product of glyphosate that accumulates in the environment [45], inhibited melanization similarly to glyphosate using G. mellonella hemolymph and a commercially available mushroom tyrosinase (Fig 1B, S1D Fig). These data show that glyphosate inhibits melanization in insects similar to what has been previously shown in fungi [26], thus indicating that glyphosate interferes with major melanin-based processes in at least 2 kingdoms of life.

\section{Glyphosate alters G. mellonella susceptibility to infection}

Next, we sought to determine whether glyphosate increased in vivo susceptibility of G. mellonella larvae to foreign organisms. We injected G. mellonella final instar larvae with $2 \mu \mathrm{g}$ of glyphosate (approximately 8 to $12 \mathrm{ng} / \mathrm{mg}$ per larvae) followed by infection with C. neoformans or a mock infection. The 2 mock infected groups, glyphosate-treated and phosphate-buffered saline (PBS)-treated, exhibited similar survival. However, in the infected groups, the glyphosate-treated larvae died faster compared to the PBS-treated controls (Gehan-Breslow-Wilcoxon test, $p=0.013$ ) (Fig 1C). A similar, but nonsignificant, trend was seen with a $C$. neoformans lac1s strain (S1E Fig). The lac1s strain is unable to produce melanin, an important virulence factor in C. neoformans pathogenesis. This strain is less virulent in the G. mellonella model [78], potentially contributing to the lack of significant differences between the glyphosate and PBS-treated groups.

The decreased survival of the glyphosate-treated group infected with C. neoformans was correlated with smaller melanized particles within nodules formed during infection (in vivo), as compared to the PBS-treated group infected with C. neoformans (Fig 1D). For these experiments, we infected and drugged G. mellonella as we do during normal infections, then collected the hemolymph 24 hours later and imaged the nodules and aggregates that formed in vivo. The PBS-treated noninfected group had smaller or virtually no melanized structures. In 2 of 3 replicates, the PBS-treated infected group had more melanized structures than the glyphosate-treated group (S1G-S1I Fig). Further, in the glyphosate-treated infected group, we observed more C. neoformans cells, and those within nodules displayed lower degrees of melanin encapsulation compared to PBS-treated larvae (chi-squared test, $p=0.0034$ ) (Fig 1E). The scoring was based on a system devised with 0 representing no melanin encapsulation of the yeast cell to 4 being the most melanin encapsulation, as depicted in Fig 1F. The one-time treatment with glyphosate did not disrupt time to larval pupation, a process mediated by laccases and phenoloxidases (S1F Fig). These data suggest a direct correlation between glyphosate treatment and increased susceptibility of G. mellonella to infection caused by decreased melaninbased immune response (nodulation).

\section{Glyphosate alters A. gambiae phenoloxidases, susceptibility to infection with malaria parasites, and survival}

To ascertain the impact of glyphosate on A. gambiae mosquito melanization, we measured the phenoloxidase activity in whole-body mosquito homogenate following the addition of glyphosate. Similar to our results with G. mellonella, glyphosate inhibited phenoloxidase activity of $A$. gambiae homogenate in a dose-dependent manner (Fig 2A). 
A.

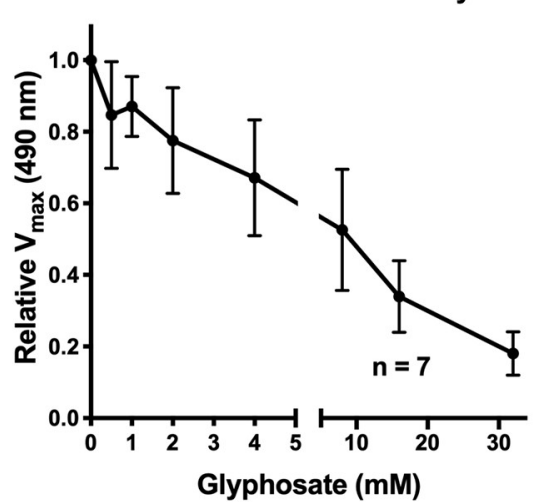

D.

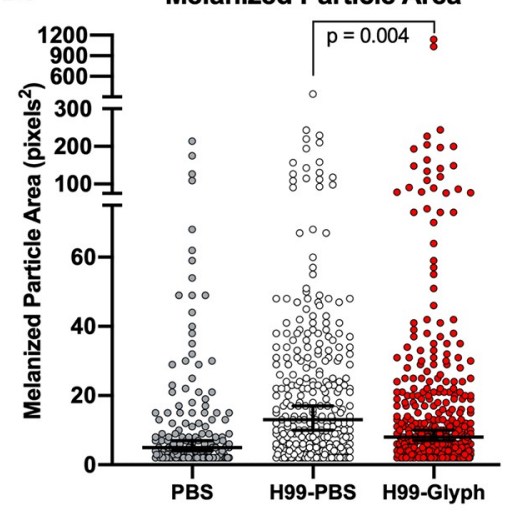

G.
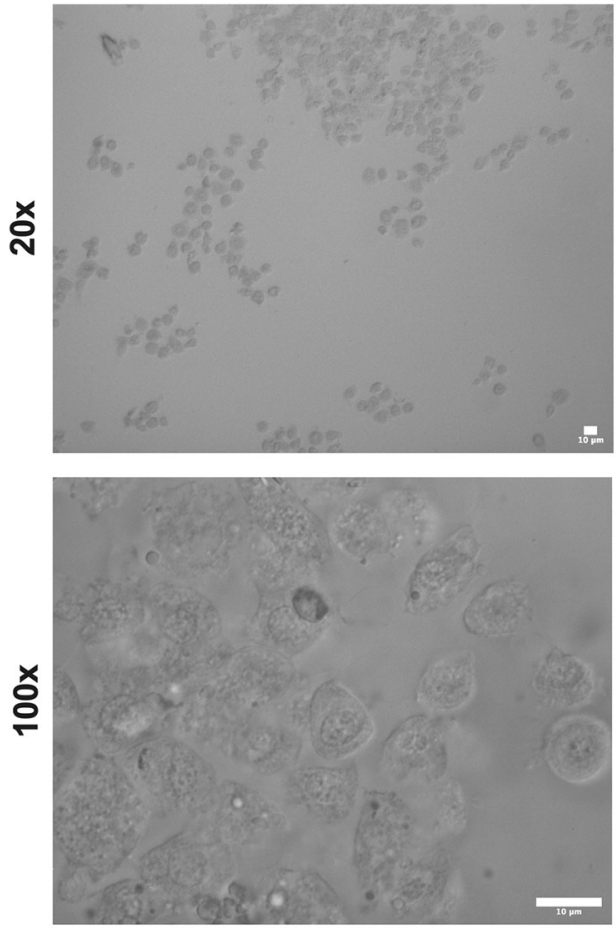

B.

G. mellonella PO Activity

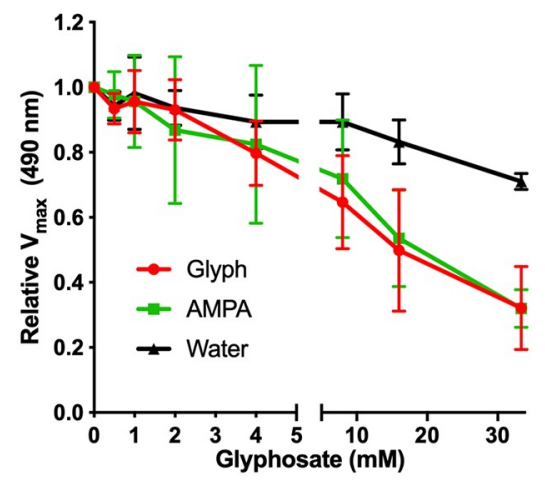

E.

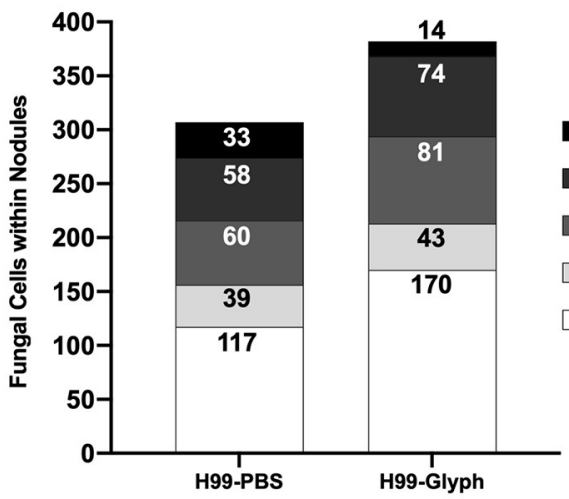

H99-PBS
C. G. mellonella Infection with C. neoformans

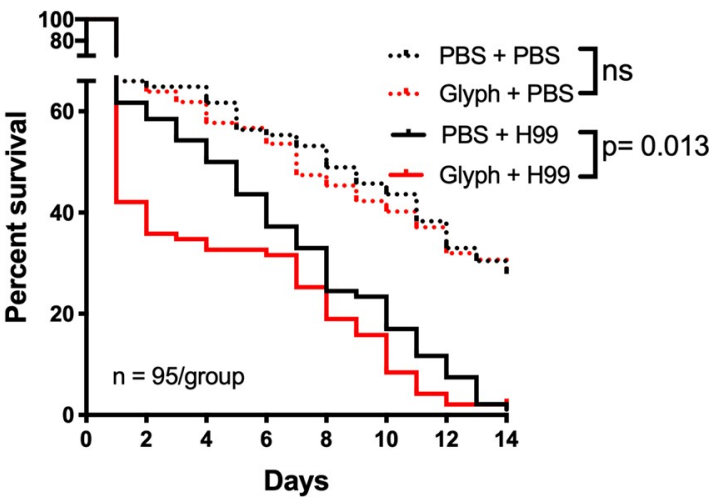

F. Representative Scoring References

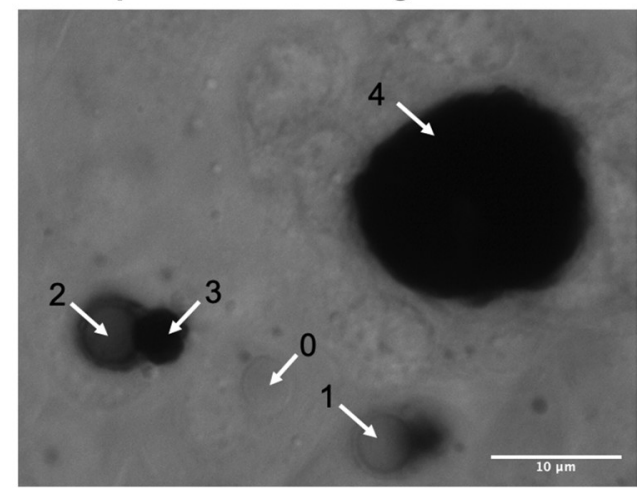

H99-Glyph
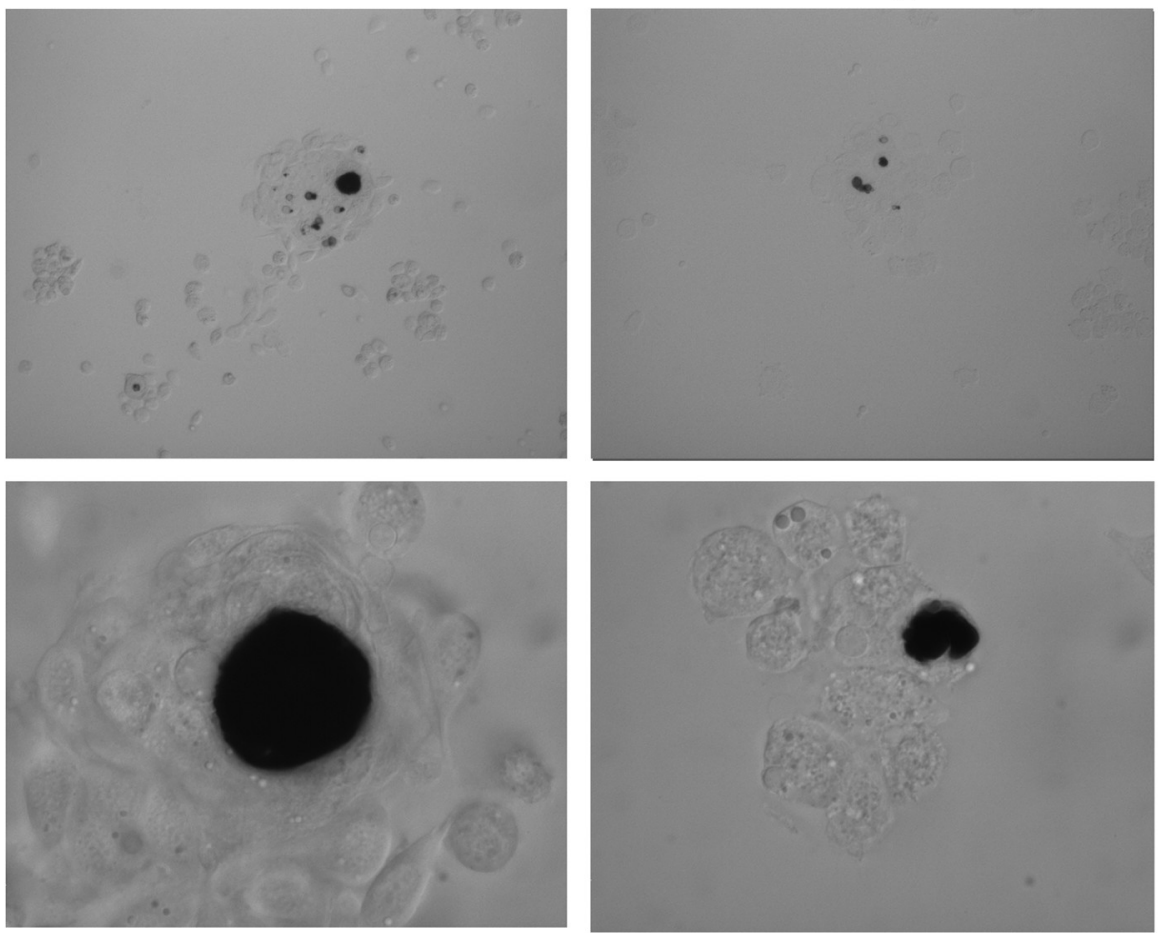
Fig 1. Glyphosate inhibits G. mellonella melanization and increases infection susceptibility. (A) Glyphosate inhibits the phenoloxidase activity of 1:10 dilutions of hemolymph without exogenously added L-DOPA. (B) AMPA, a primary metabolite of glyphosate, inhibits G. mellonella phenoloxidase-mediated melanization similar to glyphosate. Error bars in (A, B) represent \pm SD (C) G. mellonella larvae drugged with glyphosate solution $(10 \mu \mathrm{lof} 1 \mathrm{mM})$ in PBS and infected 5 hours posttreatment with $10^{4}$ cells of WT C. neoformans die rapidly compared to PBS-treated controls. Death events were recorded daily. Each infection condition represents survival of 95 animals, pooled together from 4 biological replicates, and 6 total technical replicates. Statistical significance was assessed by Gehan-Breslow-Wilcoxon test, which we used to place weight on early time points in the survival curve. We used this test because we expected to see the glyphosate-mediated differences early in the infection due to the timing of the glyphosate treatment. Since where the expected effects of the one-time pretreatment with glyphosate would be. (D) The size of the dark melanized particles within nodules upon C. neoformans infection are significantly smaller in the glyphosate-treated (10 $\mu \mathrm{l}$ of $1 \mathrm{mM})$ infected groups compared to the PBS-treated infected groups, which were analyzed for significance using a nested nonparametric Mann-Whitney-Wilcoxon rank test. Horizontal bar represents the median value and the error bars represent the $95 \%$ confidence interval (E) The degree of melanin encapsulation of the yeast within the nodule is also reduced in the glyphosate-treated (10 $\mu \mathrm{l}$ of $1 \mathrm{mM}$ ) groups, as measured on a scale of 0 (no melanin encapsulation) to 4 (very high levels of melanin encapsulation) as demonstrated in (F). Numbers in each bar represent the number of encapsulated C. neoformans for each score. Statistical significance was assessed using a chi-squared table test. Data in (D) and (E) represent data over 3 independent replicates with 3 larvae used per condition per replicate. (G) Representative brightfield micrographs showing the hemocyte and nodule formation in the different treatment groups at $20 \times$ and $100 \times$ magnification. Scale bars represent $10 \mu$ m. Nested nonparametric Mann-WhitneyWilcoxon rank test performed using R for R 4.0.2 GUI 1.72 for Mac OS at https://www.r-project.org/ (R Core Team, 2020) and the nestedRanksTest package (Version 0.2, D.G. Scofield, 2014) [77]. All other statistical analyses performed using GraphPad Prism version 8.4.3 for Mac OS, GraphPad Software, San Diego, California, USA, www.graphpad.com. For underlying data, please see Data Availability section and/or S1 Table. See also S1 Fig. AMPA, aminomethylphosphonic acid; L-DOPA, 3,4-dihydroxyphenylalanine; PO, phenoloxidase; WT, wild-type.

https://doi.org/10.1371/journal.pbio.3001182.g001

To investigate whether glyphosate rendered mosquitoes more susceptible to infection with the human malaria parasite P. falciparum, adult female mosquitoes were fed on $10 \%$ sugar solution supplemented with glyphosate at different concentrations for 5 days and then given a $P$. falciparum-infected blood meal. Parasite burden was assessed through enumeration of the Plasmodium oocyst stage at 8 days postinfection (DPI). Glyphosate-fed mosquitoes had higher oocyst burdens with an overall nonsignificant trend of increasing oocyst burden with increasing dose of glyphosate (Fig 2B). However, we observed a sharp decline in parasite burden in the $10 \mathrm{mM}$-treated group, which was likely due to the increased mortality of this group (Fig $2 \mathrm{C}$ ) resulting in few surviving mosquitoes to assess the intensity of infection. In a low $P$. falciparum infection intensity assay (S2A Fig), we observed that glyphosate-treated groups are more likely to be infected than control groups. This is important, as lower parasite burdens are more reminiscent of infections in field conditions in malaria-endemic regions [80-83].

Sugar preparations with glyphosate at environmentally relevant concentrations were given to A. gambiae mosquitoes to ascertain the herbicide's effect on the mosquito's life span. Compared to control mosquitoes (sugar-fed without glyphosate), mosquitoes given low glyphosate doses $(30$ to $300 \mu \mathrm{M})$ showed statistically significant improved survival, while those fed higher doses of glyphosate ( 1 to $10 \mathrm{mM}$ ) had equal or decreased survival, with the $10 \mathrm{mM}$ glyphosateexposed group exhibiting significantly decreased survival (Fig 2C). Additionally, we used a Cox Mixed Effects Model to account for fixed and random effects, and calculated the hazard ratios for each of the treatment groups (Fig 2D). Hazard ratios $<1$ indicate a reduced risk of death compared to the control, while hazard ratios $>1$ indicate enhanced risk of death compared to the control group. With this model, the mosquitoes treated with lower glyphosate concentrations had a hazard ratio less than 1, those treated with 1 and $3 \mathrm{mM}$ glyphosate had hazard ratios similar to 1 , and the $10 \mathrm{mM}$-treated mosquitoes had a hazard ratio significantly greater than 1 . These results suggest that glyphosate could have bimodal effects on mosquito health.

We also measured the impact of glyphosate on the mosquito cuticle and body size. There was no discernable difference in A. gambiae cuticle pigmentation after 5 days of treatment with $1 \mathrm{mM}$ glyphosate in $10 \%$ sucrose from days 3 to 8 postemergence, as measured by mean gray value of the ventral abdomen (S2C Fig). This was expected due to the typical expression of cuticular laccases being largely at the timing of pupation and the first 3 days postemergence $[23,24]$. Additionally, there were no difference in wing length, a proxy for adult size, between 


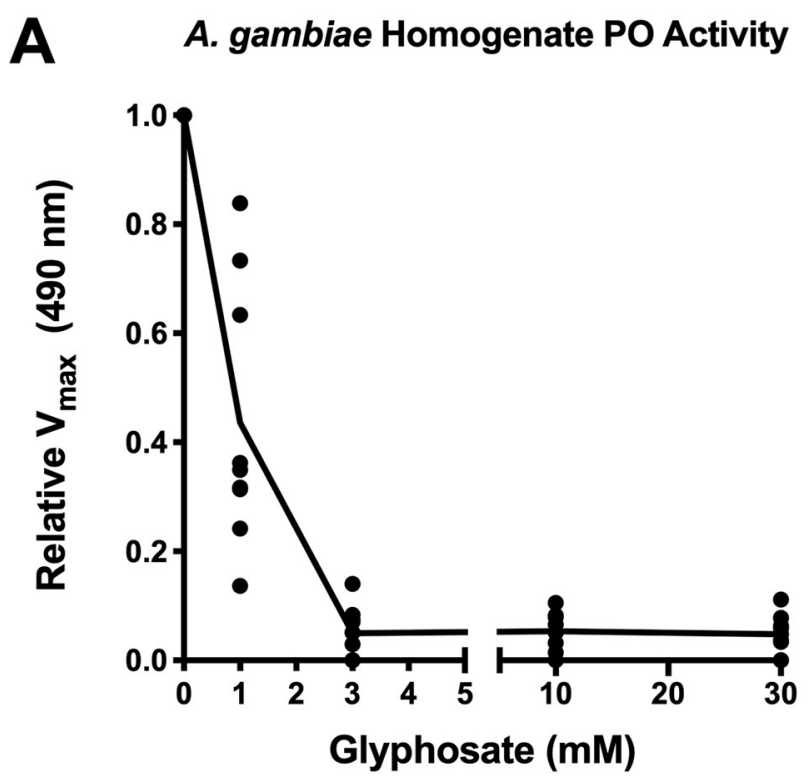

C

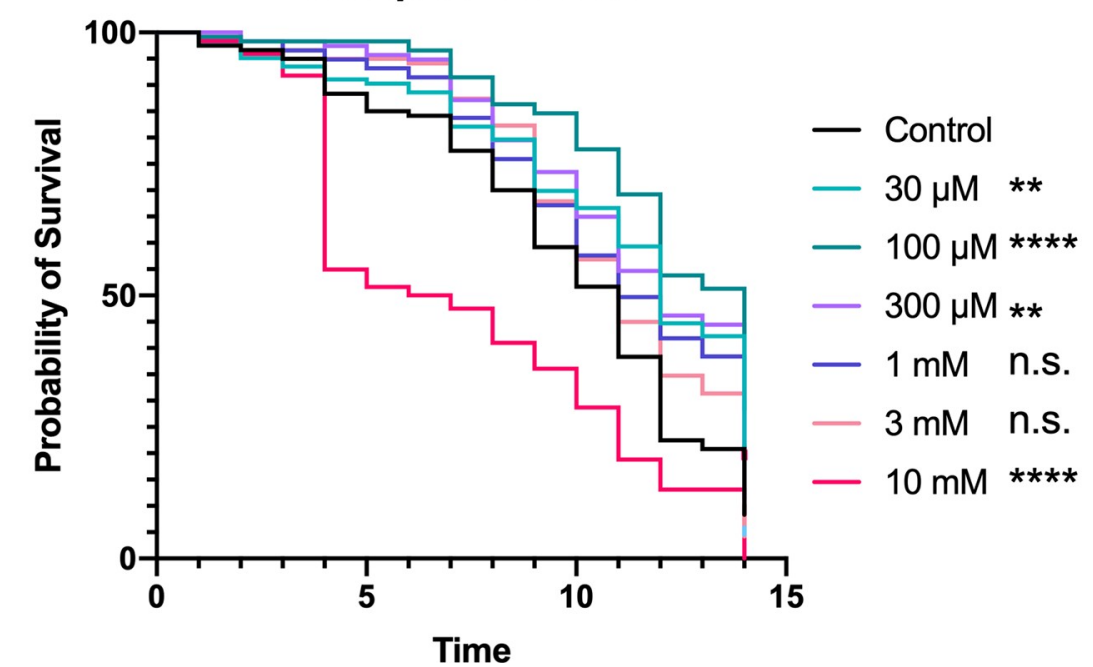

P. falciparum Infection of $A$. gambiae

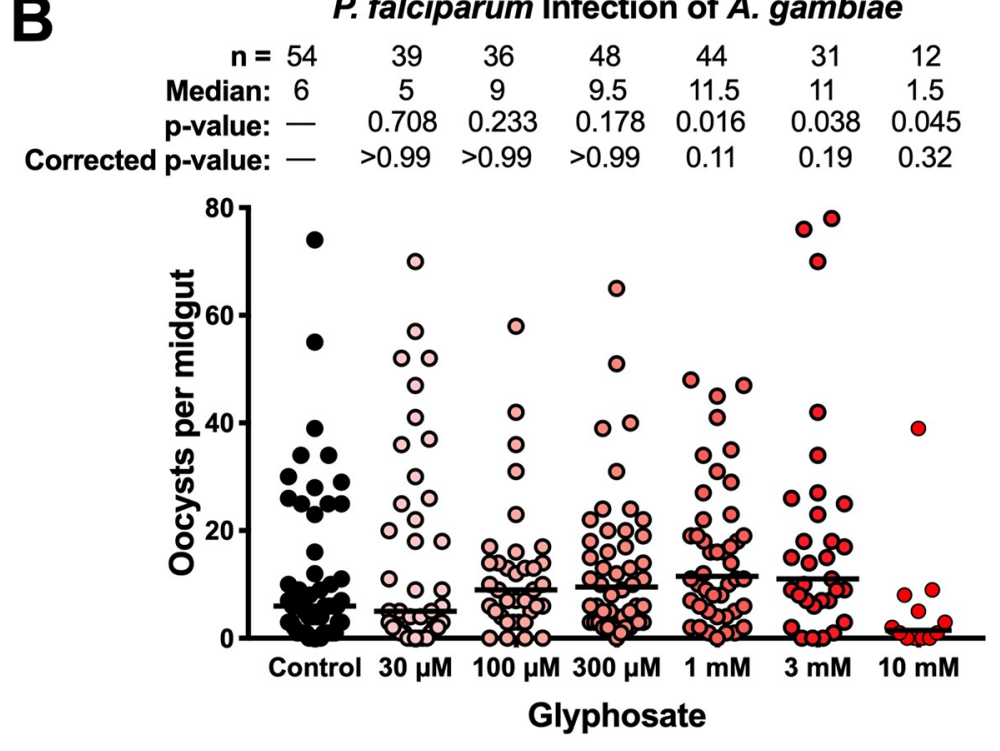

D

Cox Mixed Effect Model

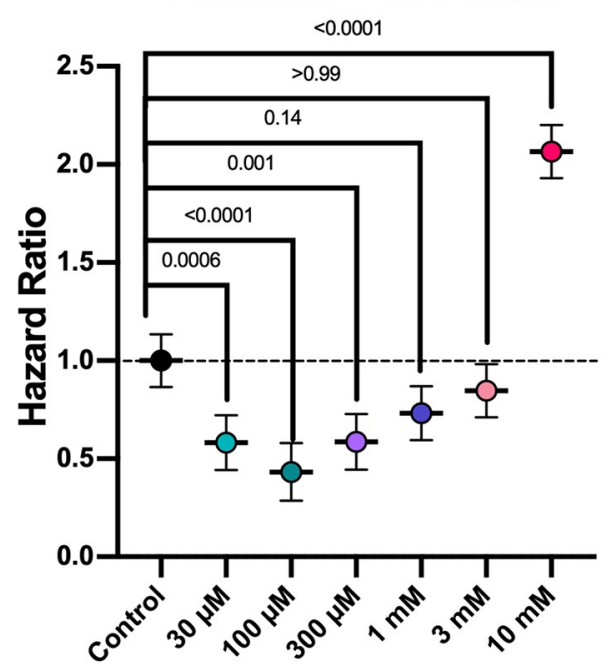

Fig 2. Glyphosate effects on A. gambiae phenoloxidase activity and susceptibility to Plasmodium infection. (A) Glyphosate inhibits phenoloxidase activity in A. gambiae homogenate. Enzyme activity represents 3 biological replicates with 3 technical replicates for each condition. (B) Glyphosate treatment increases the susceptibility of the A. gambiae to P. falciparum infection as measured by oocyst count per midgut. Increased glyphosate doses are associated with increased median oocyst burden. Parasite infection represents 4 biological replicates and 4 separate infections, line indicates median, and differences in parasite burden analyzed for significance using nonparametric Kruskal-Wallis test with each group compared to the control group with Dunn correction for multiple comparisons. (C) Low doses of glyphosate enhance the survival of adult mosquitoes, while the higher doses diminish their survival as compared to the control. Survival curves represent 120 animals from 3 independent replicates composed of groups of 40 mosquitoes, and survival was examined for statistical significance using the log-rank Mantel-Cox analysis with a Bonferoni correction for multiple comparisons. (D) Hazard ratios calculated from the Cox Mixed Effects Model to account for fixed (glyphosate treatment) and random effects (replicate). Hazard ratios $<1$ indicate lower risk of death compared to control values, and values $>1$ indicate a higher risk of death compared to the control. Hazard ratio of 1 is depicted by a dotted line. The Cox Mixed Effects modeling was performed using R for R 4.0.2 GUI 1.72 for Mac OS at https://www.r-project. org/ (R Core Team, 2020) and the coxme package (Version 2.2-16, T.M. Therneau, 2020) [79]. All other statistical analyses performed using GraphPad Prism version 8.4.3 for Mac OS, GraphPad Software, San Diego, California, USA, www.graphpad.com. The statistical significance in (C) is coded as: ns, $p>0.05 ;{ }^{*} p<0.05$; ${ }^{* *} p<0.01$; ${ }^{* * *} p<0.001$; and ${ }^{* * * *} p<0.0001$. For underlying data, please see Data Availability section and/or S1 Table. See also S2 Fig. n.s., not significant; PO, phenoloxidase.

https://doi.org/10.1371/journal.pbio.3001182.g002

the glyphosate-treated and untreated adult mosquitoes (S2D Fig). Altogether, this suggests that the observed increased parasite burden in glyphosate-treated mosquitoes cannot be explained merely by broader impacts of glyphosate on mosquito health. 


\section{Glyphosate alters the composition, but not density, of the A. gambiae midgut microbiota}

A. gambiae midgut microbiota can influence Plasmodium infection by modulating the mosquito's innate immune system and hence affecting parasite viability [84-87]. We investigated whether glyphosate had detrimental effects or influence on the A. gambiae microbiota. Colony forming unit (CFU) counts from cultures of midgut homogenates grown on LB agar demonstrated that glyphosate treatment did not affect total number of culturable gut bacteria (Fig $3 \mathrm{~A}$ ), though this method would miss any impacts on microbes that were not readily cultured by these methods. To complement our culture-dependent analysis and provide insight on microbiota community composition, we compared the total 16S rRNA composition of the midgut microbiota with and without glyphosate treatment. Glyphosate treatment altered microbiota composition, with a noted decrease in the relative abundance of Enterobacteriaceae and an increase in relative Asaia spp. populations (Fig 3B). We did not observe a dose-dependent impact on composition, as the alpha diversity (a function of the number of bacterial taxa) of mosquitos exposed to glyphosate was similar to controls (Fig 3C). However, community composition was perturbed by treatment with glyphosate, and glyphosate-treated groups and controls form 2 separate clusters in principal coordinates analysis as measured by Bray-Curtis dissimilarity (Fig 3D). These differences suggest a shift in beta diversity (prevalence of each bacterial taxon), and therefore a difference between the microbial communities of mosquitoes exposed to glyphosate versus untreated controls.

\section{Glyphosate inhibits production of dopaquinone, dopachrome, and melanin}

To understand how glyphosate inhibited melanization, we evaluated the formation of melanin intermediates in a stepwise manner using a commercially available fungal tyrosinase and the melanin precursor L-DOPA $(2 \mathrm{mM})$. Although this tyrosinase differs from insect phenoloxidase, the melanization reaction in these systems follows the same Mason-Raper pathway (Fig $4 \mathrm{~A})[88,89]$ and thus can be used to explore the mechanism of glyphosate inhibition. The first step of the reaction involves L-DOPA oxidation to dopaquinone enzymatically or spontaneously [90]. We found that glyphosate inhibited the dopaquinone production in a dose-dependent manner (Fig 4B). This inhibition was observed for both tyrosinase-mediated and autooxidation-mediated production of dopaquinone. The slopes of inhibition in the auto-oxidation and tyrosinase-mediated oxidation were similar. This indicated that the tyrosinase reaction dopaquinone levels would remain unchanged by glyphosate treatment if the inhibition of "background" auto-oxidation dopaquinone production were taken into consideration. These results suggested that dopaquinone inhibition was primarily rooted in preventing the oxidation of L-DOPA independent of tyrosinase.

Dopaquinone spontaneously cyclizes to form cyclodopa, which then undergoes a redox exchange with another dopaquinone molecule to form 1 molecule of dopachrome and 1 reformed molecule of L-DOPA. Dopachrome is a pink-orange melanin intermediate that has an absorbance maximum at $475 \mathrm{~nm}$. Dopachrome is a useful proxy product for tyrosinase-mediated reaction kinetics and evaluating the melanization reactions and redox exchange [91]. The rate of dopachrome formation and the amount of dopachrome produced were determined by measuring changes in absorbance during a reaction between L-DOPA and tyrosinase. There was a strong dose-dependent inhibition of dopachrome formation with glyphosate (Fig 4C), implying that the compound's inhibitory effects were upstream of dopachrome.

We tracked the reaction over 5 days to confirm inhibition of melanin synthesis. Glyphosate inhibited the production of a black pigment dose-dependently, as measured by the absorbance 


\section{A Glyphosate and Mosquito Microbiome}

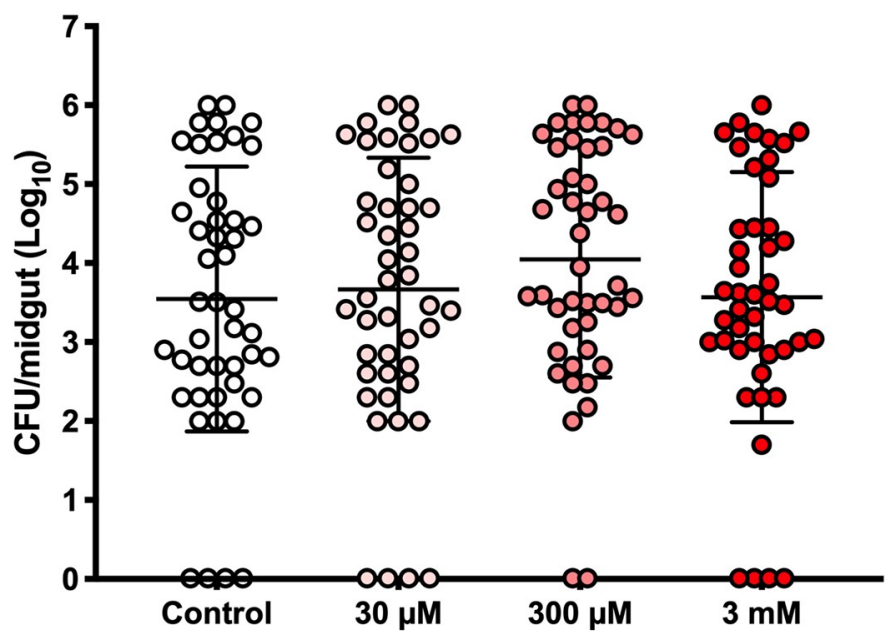

B

Glyphosate
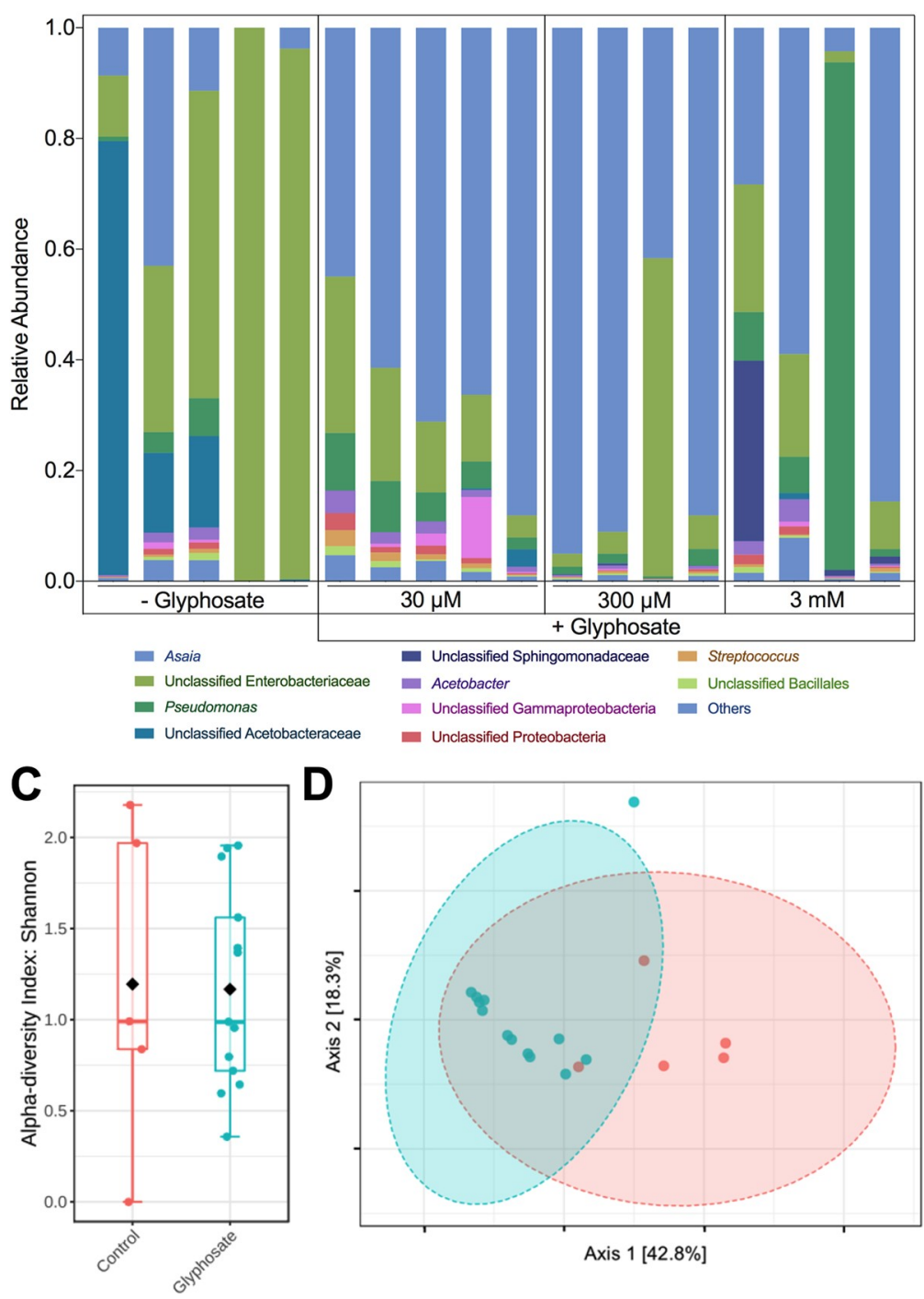

D

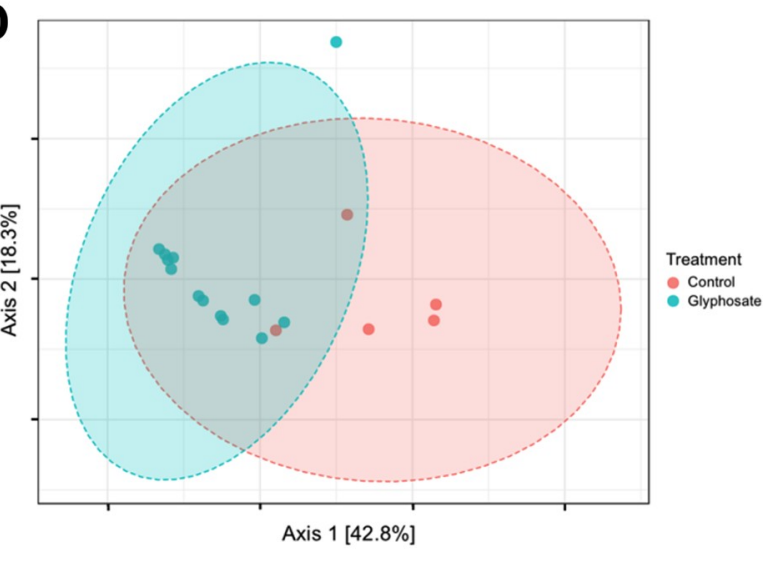


Fig 3. Glyphosate alters the composition, but not density, of the A. gambiae midgut microbiota. (A) Glyphosate does not alter microbial density of the culturable mosquito midgut bacteria (grown on LB agar). Each sample consists of 40-50 individual mosquito midguts over 3 independent replicates. Error bars represent the mean and \pm SD. (B) Glyphosate alters the composition of the mosquito microbiota, leading to decrease of Entereobacteriacae and an increase of Asaia spp. (C) The glyphosate treatments do not significantly alter alpha diversity as measured by the Shannon Index (statistical analysis conducted using one-way ANOVA; NS $=p>0.05$ ). (D) However, the glyphosatetreated and control-treated microbiota form distinct clusters in principle coordinates analysis, measured by BrayCurtis dissimilarity. Statistical significance was tested by PERMANOVA $(p<0.001, \mathrm{R}=0.557)$. Each treatment group represents 5 individual mosquito midguts. For underlying data, please see Data Availability section and/or S1 Table. For more information, see also S3 Fig. CFU, colony forming unit; n.s., not significant.

https://doi.org/10.1371/journal.pbio.3001182.g003

A<smiles>CC(C)(C)Oc1cc2c(cc1O)CC(C(=O)O)N2</smiles>

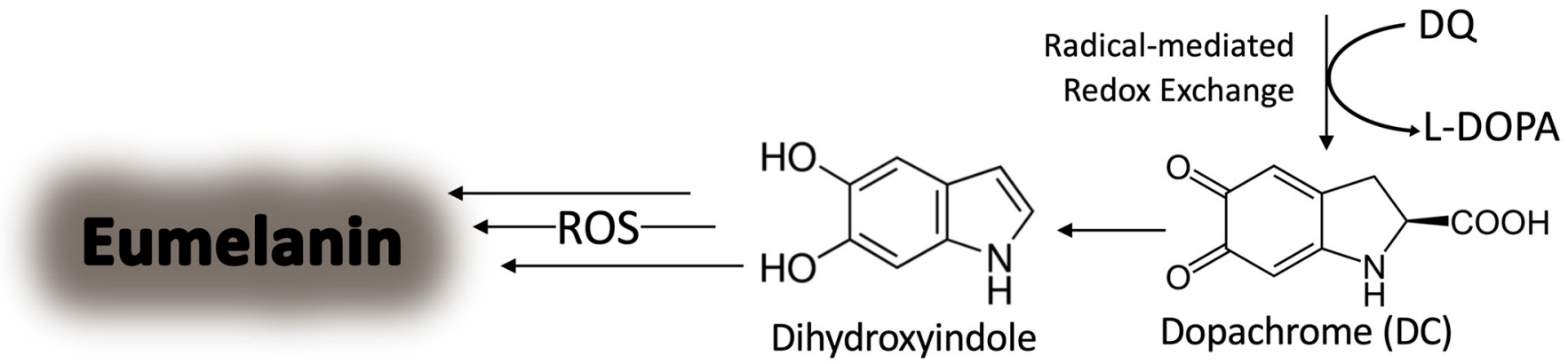

B

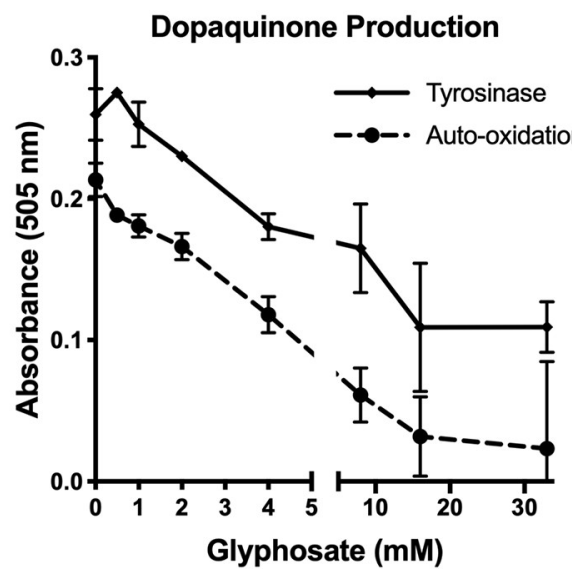

C

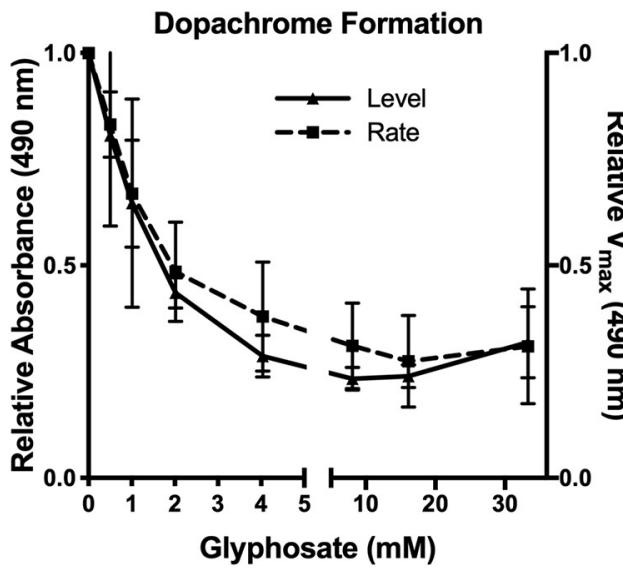

D

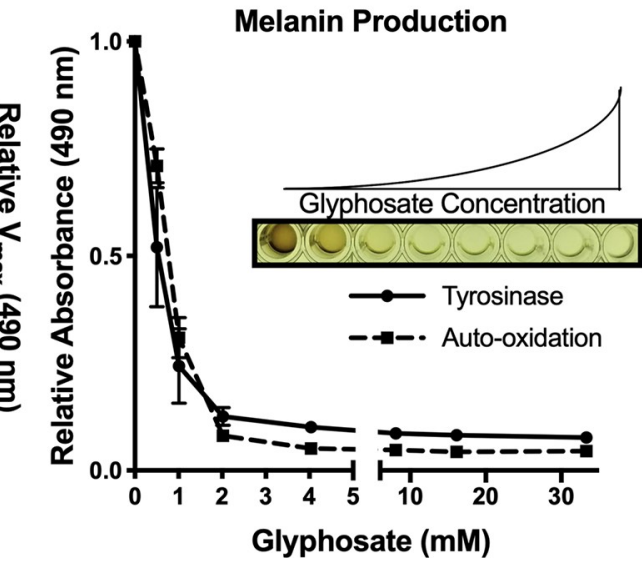

Fig 4. Glyphosate inhibits in vitro melanin production. (A) An overall schematic of the Mason-Raper pathway of melanization mediated by tyrosinase and auto-

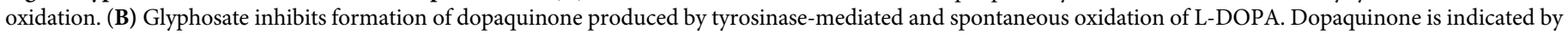
the absorbance of an MBTH-dopaquinone adduct pigment at $505 \mathrm{~nm}$. Absorption levels are shown relative to the no glyphosate control with background (MBTH

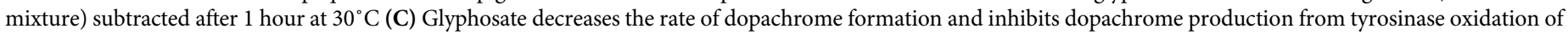

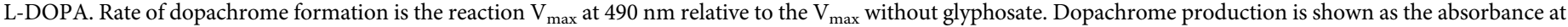

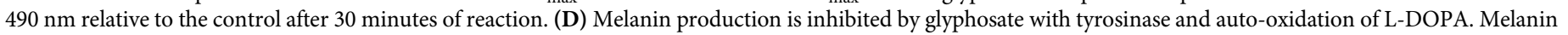
levels are measured as the absorbance at $490 \mathrm{~nm}$ after 5 days of reaction. Inset shows a representative image of the data in (D) showing melanization inhibition with increasing glyphosate concentration. Values are depicted relative to the no glyphosate control. Error bars represent \pm SD. Each experiment was performed at least 3 independent replicates. For underlying data, please see Data Availability section and/or S1 Table. L-DOPA, 3,4-dihydroxyphenylalanine; MBTH, 3-methyl2-benzothiazoninone hydrazone; ROS, reactive oxygen species. 
of the tyrosinase reaction on day 5 (Fig 4D). Interestingly, glyphosate also inhibited the formation of pigment that derives from auto-oxidation of L-DOPA (Fig 4D). This implies that glyphosate inhibited pigment production nonenzymatically.

\section{Phosphate-containing compounds inhibited melanization similarly to glyphosate}

To gain insight into the chemical features of glyphosate that inhibited melanogenesis, we assayed several structurally similar compounds using the same in vitro mushroom tyrosinase assay. To test the effect of the amino acid functional group, we compared glyphosate alongside its non-phosphate analog, glycine. We also tested the inhibitory effects of phosphoserine and serine on melanin production. Phosphoserine inhibited dopaquinone, dopachrome, and melanin formation to nearly the same extent as glyphosate (Fig 5A-5C). In contrast, neither glycine nor serine inhibited dopaquinone, dopachrome, or overall melanin formation (Fig 5A-5C). We tested the inhibitory effects of other phosphate-containing compounds including organophosphates (phosphonoacetic acid), phosphoesters (pyrophosphate), and phosphoric acid. All of the phosphate-containing compounds inhibited dopaquinone production (Fig 5A) and dopachrome formation (Fig 5B) in a manner nearly identical to glyphosate, but differed slightly from each other in melanin inhibition (Fig 5C).

Similar to glyphosate, these compounds all inhibited auto-oxidation of L-DOPA comparably to their inhibition of tyrosinase-mediated melanin production (Fig 5E). This further illustrates that glyphosate and similar phosphate-containing compounds inhibit melanin in a nonenzymatic fashion. These data suggest that the phosphate functional groups of these compounds may be responsible for the melanin-inhibitory properties.

\section{Glyphosate does not react with L-DOPA or inhibit tyrosinase directly}

We considered the possibility that glyphosate inhibited melanogenesis and dopaquinone production by reacting with the L-DOPA substrate. To measure the reaction between these compounds, we analyzed mixtures of L-DOPA and glyphosate by ${ }^{1} \mathrm{H}-\mathrm{NMR}$ and ${ }^{31} \mathrm{P}-\mathrm{NMR}$. We found no evidence of interaction between the 2 compounds based on peak shifts of hydrogen and phosphorous at both high (60 mM glyphosate and $20 \mathrm{mM} \mathrm{L-DOPA})$ and low concentrations ( $6 \mathrm{mM}$ glyphosate and $5 \mathrm{mM} \mathrm{L-DOPA)} \mathrm{(S4} \mathrm{Fig).}$

If glyphosate was inhibiting melanin production through the formation of a covalent bond with tyrosinase, the inhibition should be irreversible. To test this, we treated $20 \mu \mathrm{g} / \mathrm{ml}$ tyrosinase with $5.63 \mathrm{mg} / \mathrm{ml}(33.33 \mathrm{mM})$ glyphosate and removed the glyphosate by dialysis. The glyphosate-treated enzyme had similar activity to the control (Fig 6A), making a strong case against a mechanism whereby glyphosate inhibited melanogenesis through irreversible inhibition of tyrosinase. Instead, analysis of the tyrosinase reaction by Michaelis-Menten kinetics assay with L-DOPA and glyphosate suggested that glyphosate is a noncompetitive inhibitor of melanin and dopachrome production (Fig 6B). Further, we tested tyrosinase activity as a function of enzyme concentration with and without glyphosate and constant concentration of L-DOPA. We found that the slope of the glyphosate-treated enzyme is lower than the watertreated control (Fig 6C). This indicates that glyphosate-mediated inhibition is reversible [92,93]. Given that our findings showed that glyphosate inhibits auto-oxidation and tyrosinase-mediated oxidation, we believe that the reversible inhibition is due to glyphosate interfering with the L-DOPA substrate's ability to be oxidized rather than the enzyme's ability to oxidize. This could be represented by the following where $E$ represents tyrosinase, $S$ represents 
A

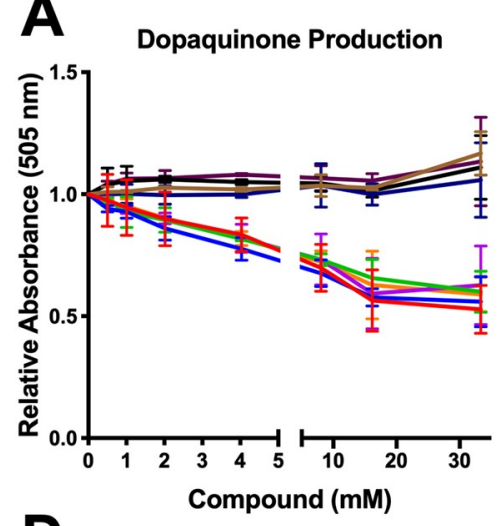

D

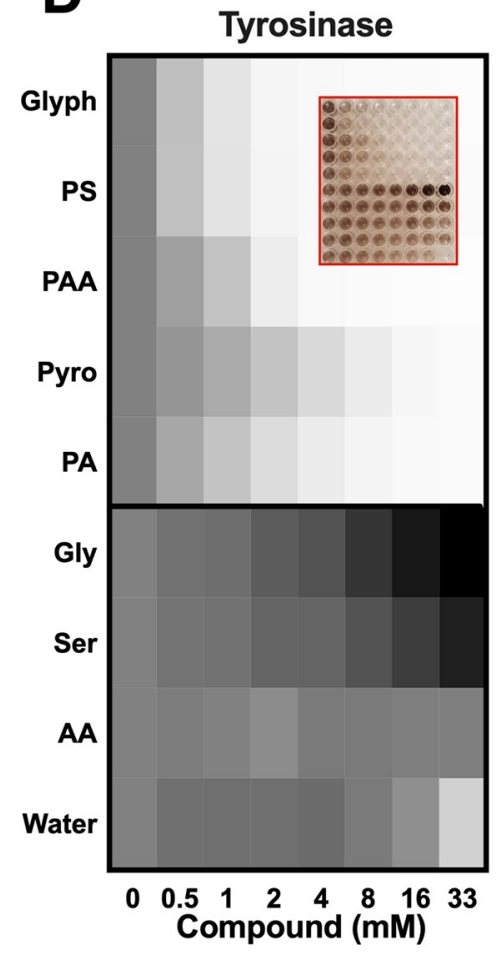

B

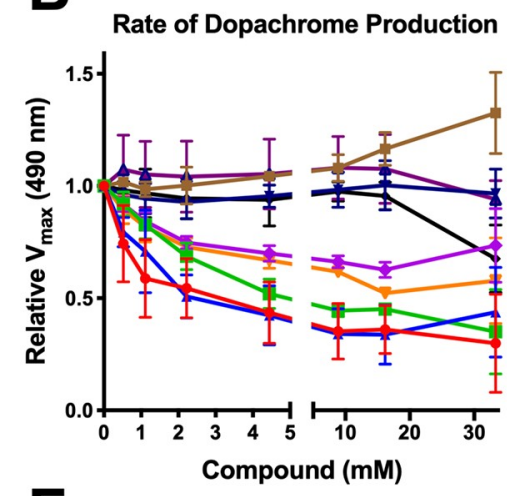

E

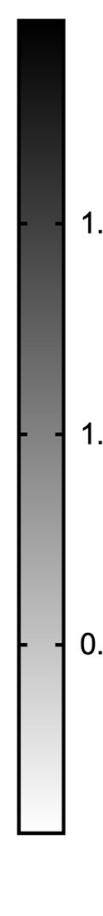

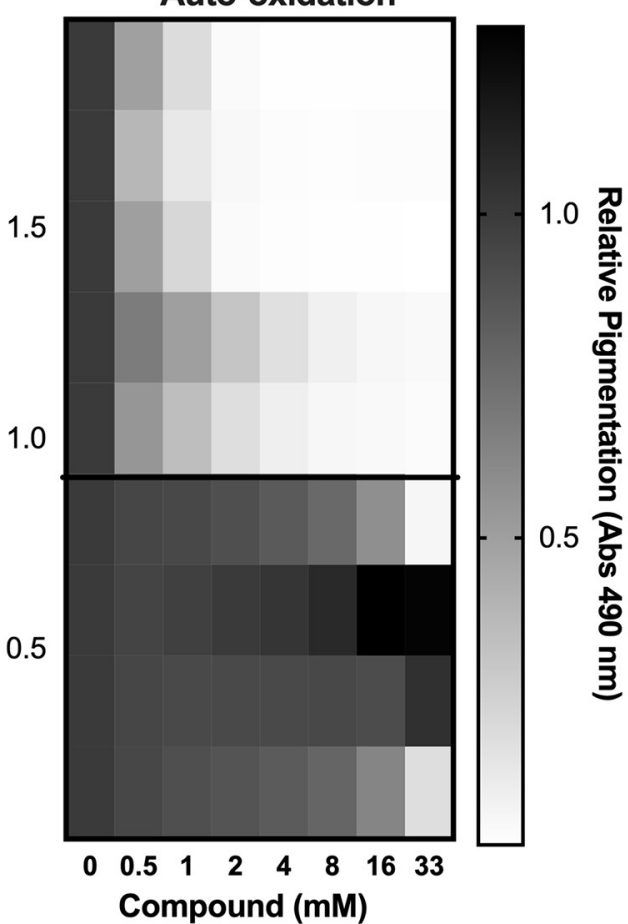

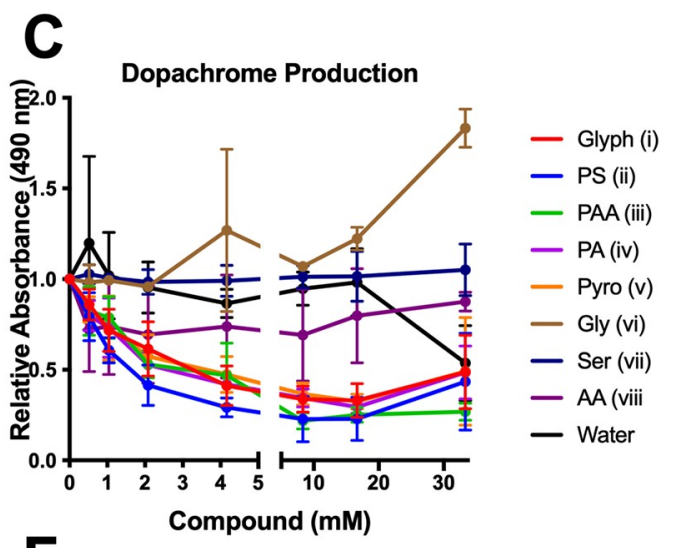

F i.<smiles>O=C(O)CNCP(=O)(O)O</smiles>

Glyphosate ii.<smiles>NC(COP(=O)(O)O)C(=O)O</smiles>

Phosphoserine iii.<smiles>CCOP(=O)(O)OP(=O)(O)OC</smiles>

Phosphoacetic Pyrophosphate Acid

vii.<smiles>NCC(=O)O</smiles>

Glycine v.<smiles>O=P(O)(O)O</smiles>

Phosphoric Acid

viii.<smiles>NC(CO)C(=O)O</smiles><smiles>CC(=O)O</smiles>

Acetic Acid

Fig 5. Phosphate-containing compounds inhibited melanization similarly to glyphosate. Glyphosate, PS, PAA, pyro, and PA inhibit dopaquinone formation (A), rate of dopachrome formation (B) and dopachrome levels (C), and melanin formation (D), whereas their respective non-phosphate analogs, gly, ser, and AA do not inhibit any step of melanization (A-D). (E) Auto-oxidation of L-DOPA is inhibited by glyphosate, PS, PAA, pyro, and PA in a similar manner. The compounds tested (F) were diluted in $300 \mathrm{mM}$ stock solution and titrated to $\mathrm{pH}$ between 5 and 6 . Absorption and rates are shown relative to the internal no drug control. Grayscale bars represent mean absorbance at $490 \mathrm{~nm}$ relative to no compound control. The darker colors correspond to increased pigment formation. Inset shows a representative image of the data in (D) showing the effects of the compounds on melanization. Error bars in (A-C) represent \pm SD. Each experiment represents at least 3 independent replicates. For underlying data, please see Data Availability section and/or S1 Table. AA, acetic acid; gly, glycine; L-DOPA, 3,4-dihydroxyphenylalanine; PA, phosphoric acid; PAA, phosphonoacetic acid; PS, $o$-phosphoserine; pyro, pyrophosphate; ser, serine.

https://doi.org/10.1371/journal.pbio.3001182.g005

L-DOPA, $I$ represents glyphosate, and $P$ represents dopaquinone/melanin:

Normal Enzymatic Reaction : $E+S \rightleftharpoons E S \longrightarrow P$

Inhibited Enzymatic Reaction : $E+S+I \rightleftharpoons E+S I \dashv P$ 
A

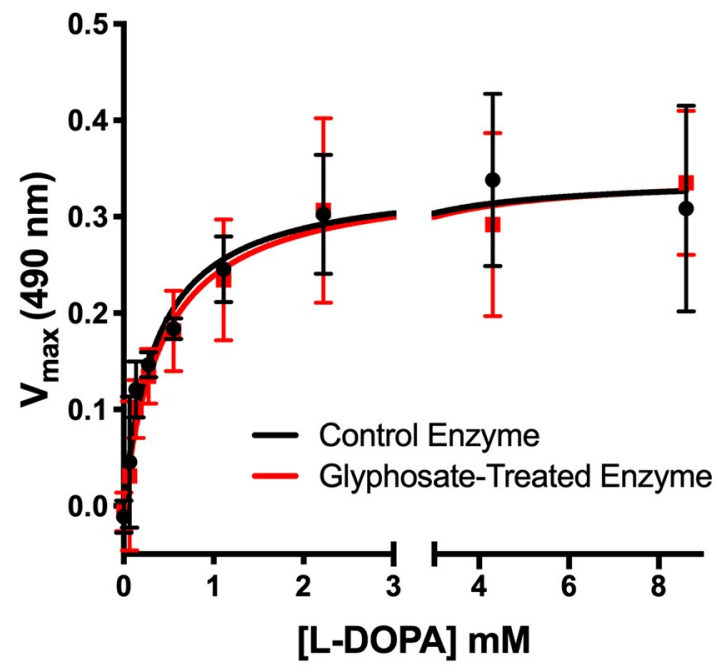

C

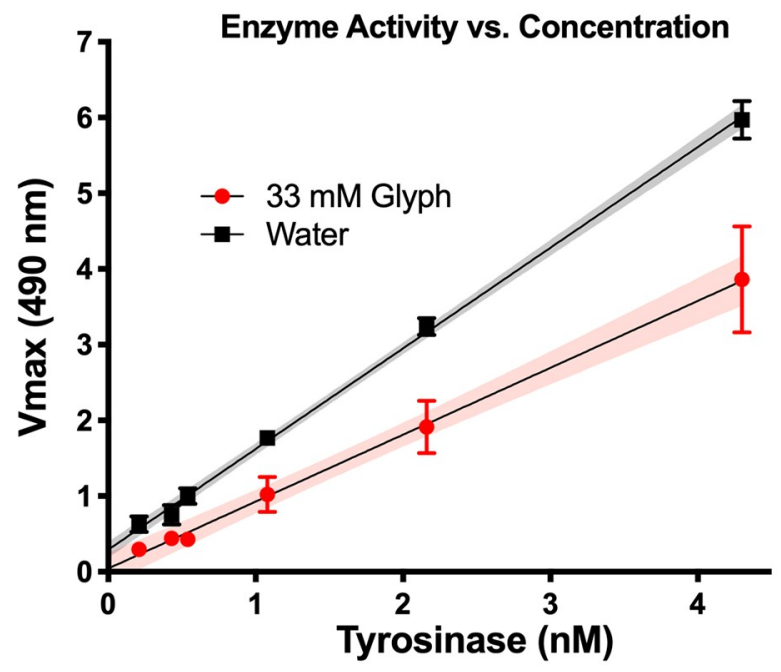

B
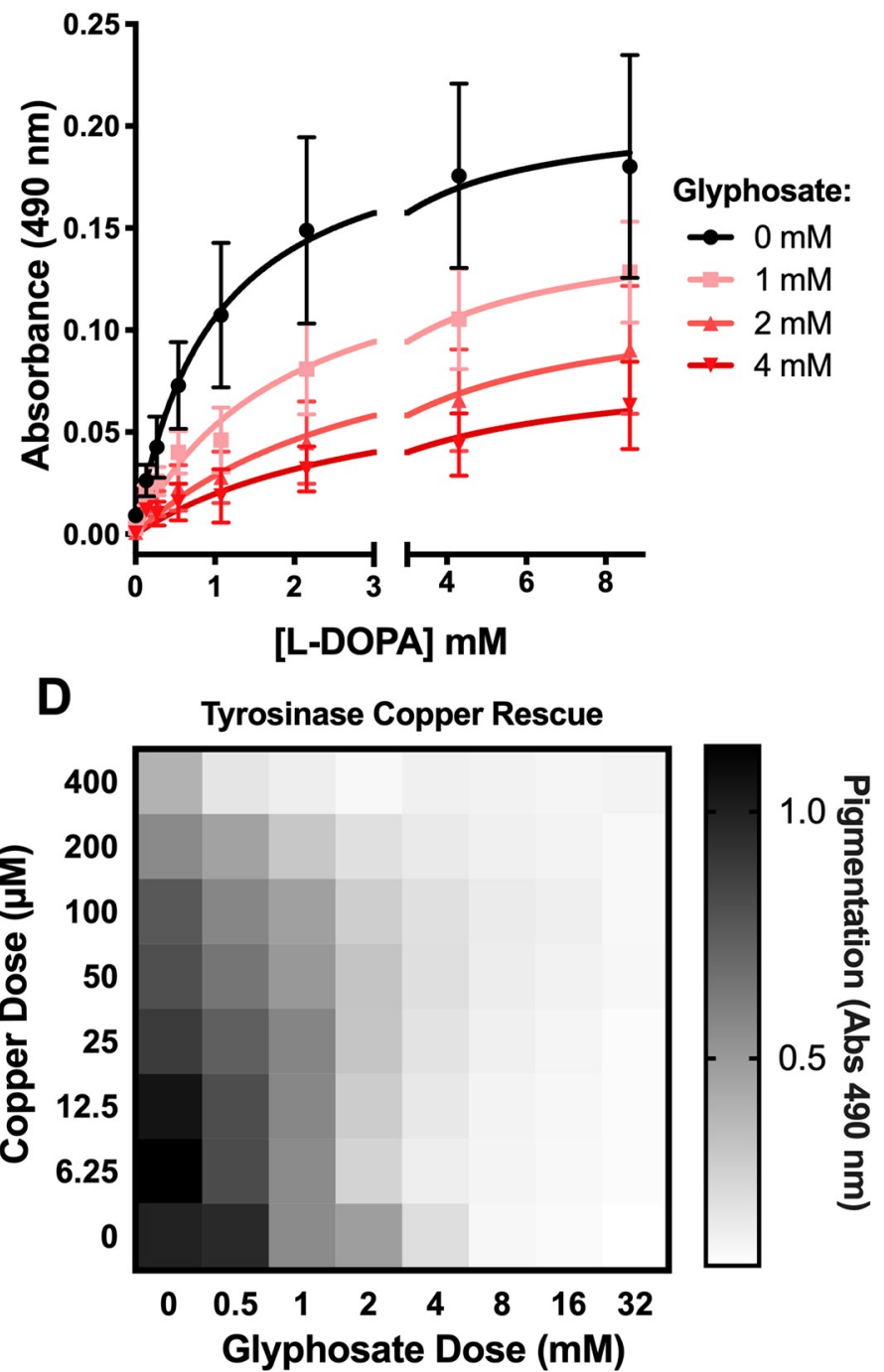

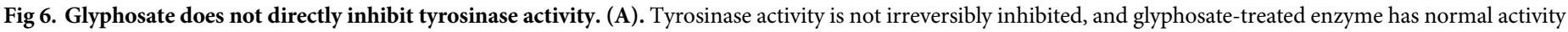
when glyphosate is dialyzed out of solution. (B) Glyphosate appears as a noncompetitive inhibitor of tyrosinase in Michaelis-Menten kinetics assays measuring the

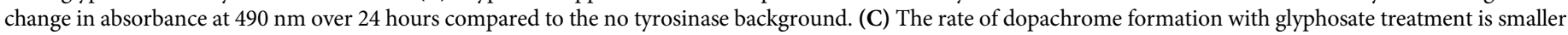

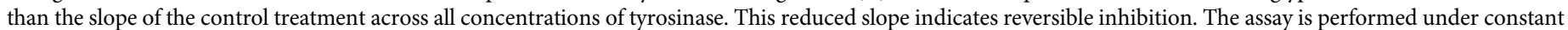

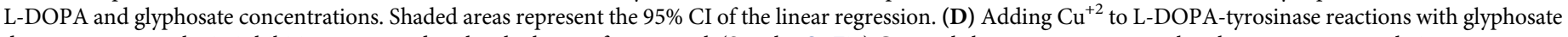
does not rescue melanin inhibition compared to the glyphosate-free control. (See also S5 Fig) Grayscale bars represent mean absorbance at 490 nm relative to no glyphosate and no copper control. The darker colors correspond to increased pigment formation. Error bars in (A-C) represent \pm SD. Each experiment represents at least 3 independent replicates. For underlying data, please see Data Availability section and/or S1 Table. L-DOPA, 3,4-dihydroxyphenylalanine.

https://doi.org/10.1371/journal.pbio.3001182.g006

Copper ions are important for tyrosinase activity. Since glyphosate is a metal chelator, [94,95], we evaluated whether glyphosate's inhibitory effect was due to this property. We added copper ions to the L-DOPA and tyrosinase reaction to rescue the glyphosate inhibition. We performed the experiment with 8 concentrations of copper (II) sulfate for each of the 8 glyphosate concentrations. In general, the addition of copper did not rescue the glyphosate dependent inhibition of melanin (Fig 6D). However, low concentrations of copper (6.25 to $25 \mu \mathrm{M}$ ) increased tyrosinase activity, while high concentrations of copper (50 to $400 \mu \mathrm{M}$ ) reduced activity, indicating low copper can boost enzyme activity, while higher concentrations 
inhibit the reaction. However, this hormesis-like effect was not observed at increasing glyphosate concentrations (S5 Fig). This result indicates that glyphosate's ability to chelate copper ions could have a protective effect in high copper environments, which would otherwise lead to negative effects on enzymatic activity and other biological processes. Similar results have been previously seen in Eisenia fetida earthworms exposed to high copper conditions in soil [96]. Glyphosate contamination of copper-rich soil reduced the detrimental effects of the metal's toxicity, presumably due to the glyphosate's copper chelation properties [96].

\section{Glyphosate affects the oxidative properties of melanogenesis}

Melanogenesis is dependent on the spontaneous radicalization of quinone intermediates [97]. Dopaquinone radicals and cyclodopa undergo a radical-mediated redox exchange that converts cyclodopa into dopachrome and dopaquinone into L-DOPA. Further downstream, ROS catalyze the polymerization of dihydroxyindole into eumelanin. Glyphosate's inhibitory effect could be due to a role as a free-radical scavenger or antioxidant. Since the inhibitory compounds blocked spontaneous oxidation of L-DOPA (Fig 5E), they are antioxidants. To measure this radical-quenching ability, we used a 2,2'-azino-bis(3-ethylbenzothiazoline-6-sulfonic acid) (ABTS) assay in which ABTS radicals are blue, but when quenched, the solution becomes colorless. The degree of discoloration is a proxy for radical concentration and antioxidant strength. Glyphosate quenched the ABTS radical to some degree, but only after several hours of reaction (S6A Fig), which did not occur with the other inhibitory phosphate group containing compounds evaluated (Fig 7A). This indicates that direct free-radical scavenging may not be the primary mechanism of melanin inhibition for glyphosate.

Phosphoric acid is a well-known synergist that boosts the antioxidant properties of phenolic compounds. Phosphoric acid, and other synergists such as citric acid, malic acid, and tartaric acid do not directly quench free radicals themselves, but instead work by regenerating antioxidants, thus becoming "sacrificially oxidized," or chelating metal ions in solution $[98,99]$. Alternatively, glyphosate could be reacting with existing antioxidants to strengthen and/or regenerate them into "active" form. In this instance, the glyphosate would be bolstering the antioxidant properties of L-DOPA.

We observed that the synergist citric acid inhibited melanization similarly to glyphosate and phosphoric acid (Fig 7B and 7C). The addition of glyphosate, phosphoserine, and phosphoric acid enhanced the antioxidant properties of L-DOPA in an ABTS assay in a similar manner as citric acid (Fig 7D). This suggests that glyphosate may act as an inhibitor via antioxidant synergism. The synergy is the ratio of the quenching capacity of the L-DOPA and the compounds alone to the quenching capacity of L-DOPA combined with the compound. The lower this ratio, the more synergistic the compounds are with L-DOPA (S6B Fig). These values indicate that the inhibitory compounds are synergistic, whereas the noninhibitory glycine and serine are not as synergistic.

The inhibition of melanin was independent of the L-DOPA to glyphosate ratio, and glyphosate's $\mathrm{IC}_{50}$ is approximately $1 \mathrm{mM}$ regardless of L-DOPA concentration (S7 Fig). This could be explained by a general antioxidant effect on solution.

\section{Glyphosate alters the oxidation-reduction potential of the system}

L-DOPA is a more effective antioxidant when it is oxidized or radicalized and has a better ability to form adducts with other radicals [100]. Since glyphosate is acting as a synergistic antioxidant, it may be driving L-DOPA oxidation and possibly radicalization in which L-DOPA scavenges radicals better. This has the potential to disrupt melanin synthesis by stopping the spontaneity of redox exchange and dopaquinone formation. 
A

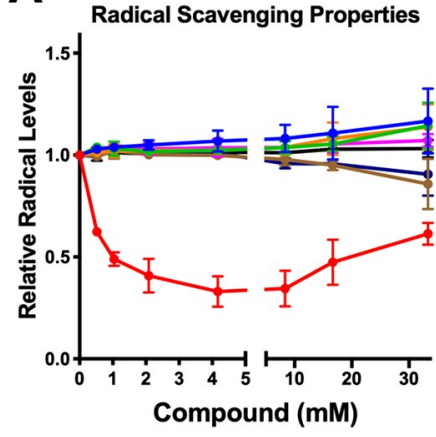

C

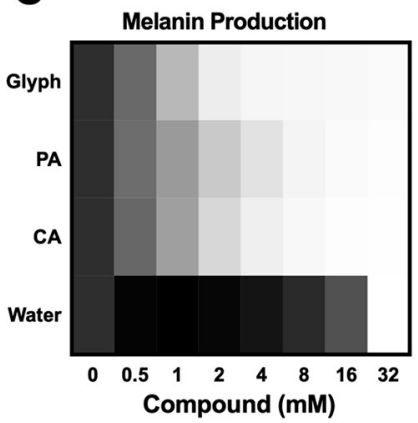

E

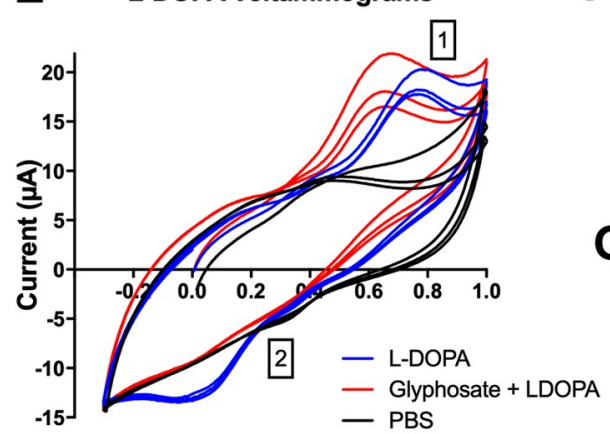

Potential applied (V)

H Dose Dependent Effect of Glyphosate

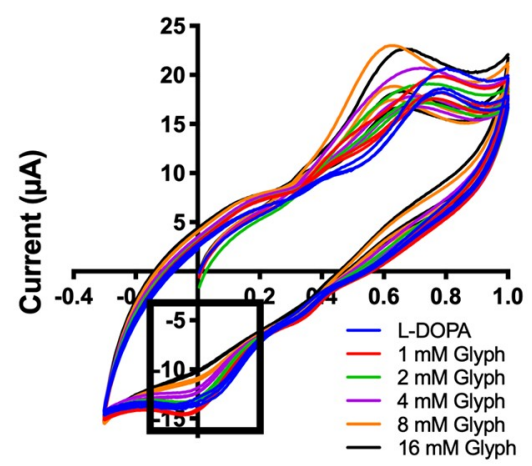

Potential applied (V)
B

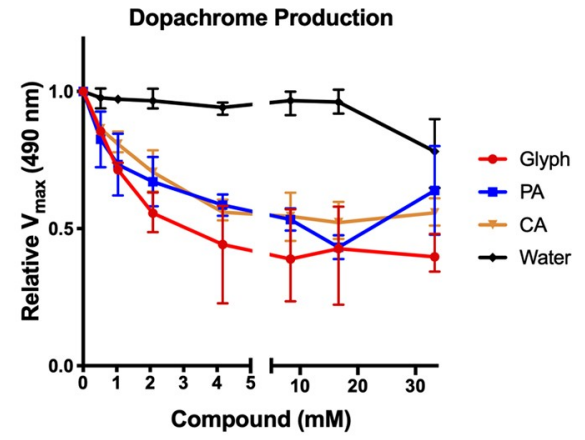

D

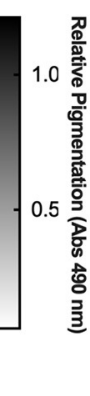

Acid Synergism With L-DOPA

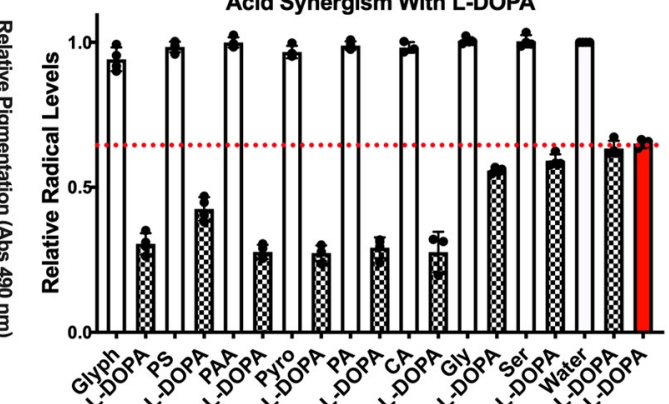

F Peak 1: L-DOPA Oxidation

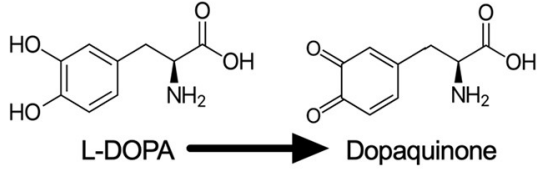

G Peak 2: Dopaquinone Reduction<smiles>N[C@@H](CC1=CC(=O)C(=O)C=C1)C(=O)O[Na]</smiles> 
Fig 7. Glyphosate affects the oxidative properties of melanogenesis. (A) None of the melanin inhibitors exhibit radical quenching properties in an ABTS assay aside from glyphosate, which shows weak antioxidant properties after several hours in the ABTS solution. Absorbance at $734 \mathrm{~nm}$ is an indicator of how much ABTS remains in radical form (not quenched). (B, C) Citric acid (CA), a nonradical quenching antioxidant (antioxidant synergist), exhibits similar melanin inhibition as glyphosate and phosphoric acid, another known antioxidant synergist. Grayscale bars in (C) represent absorbance at $490 \mathrm{~nm}$ relative to no compound control, with the darker colors correspond to increased pigment formation. (D) Glyphosate, phosphoserine, phosphoric acid, and citric acid show synergy with the antioxidant L-DOPA. The addition of these compounds to L-DOPA enhances its radical quenching abilities by approximately $50 \%$. Black dotted line represents the normalized ABTS absorbance treated with water. The other compounds tested here alone do not show much deviation from this line. The blue dotted line indicates the ABTS solution treated with L-DOPA alone. ABTS treated with L-DOPA and synergetic compounds together are below this line. (E) Average cyclic voltammogram showing the changes in oxidation and reduction of L-DOPA and dopaquinone when exposed to $16 \mathrm{mM}$ glyphosate but not water. Numbers correspond to shifted peaks or peaks with less current compared to the water control. Peak 1 corresponds to L-DOPA oxidation (F); Peak 2 likely corresponds to dopaquinone reduction $(\mathbf{G})$. Glyphosate shifts Peak 1 and 2 toward a decreased redox potential and diminishes the current of Peak 1 and 2 in a dose-dependent manner $(\mathbf{H})$, notably decreasing Peak 2 current intensity to the point of nonexistence (I). Each experiment represents at least 3 independent replicates. Error bars in $(\mathbf{A}, \mathbf{B}, \mathbf{D})$ represent $\pm S D$. For underlying data, please see Data Availability section and/or S1 Table. See also S6 and S8 Figs. CA, citric acid; Glyph, glyphosate; L-DOPA, 3,4-dihydroxyphenylalanine; PA, phosphoric acid; PAA, phosphonoacetic acid; PS, ophosphoserine; Pyro, pyrophosphate; Ser, serine.

https://doi.org/10.1371/journal.pbio.3001182.g007

To investigate whether the addition of glyphosate changed the oxidation properties of L-DOPA, we used cyclic voltammetry-a technique to measure the electrochemical properties of solutions and previously used to study quinone electrochemistry [101,102]. Voltammetry performed on L-DOPA solutions with glyphosate showed dose-dependent shifts towards a negative potential (Fig 7E and 7H) in peaks that corresponded to L-DOPA oxidation [103] (Peak 1). We validated these as L-DOPA oxidation peaks by performing voltammetry on various L-DOPA concentrations (S8A Fig). The peak shift towards negative potentials indicates the L-DOPA was oxidized more easily and had less ability to be an oxidant, similar to the negative potential shifts associated with alkaline $\mathrm{pH}$ and increased oxidation [104]. We controlled for any $\mathrm{pH}$-dependent peak shifts by adjusting each solution to $\mathrm{pH} 6.00$ prior to measurement. Decreased oxidizing power can lead to significant effects, as melanin biosynthesis is reliant upon catechol oxidation and high redox potentials of quinones. Interestingly, with increased glyphosate, the L-DOPA solution had a lower current intensity associated with the reduction of dopaquinone to L-DOPA (Peak 2). In cyclic voltammetry, smaller peaks indicate that less of the compound is oxidized or reduced. The decreased Peak 2 current became virtually nonexistent with increasing glyphosate concentrations (Fig 7E, 7H, and 7I). This implies that dopaquinone, represented by Peak 2, is either not being formed during L-DOPA oxidation or cannot be reduced back into L-DOPA. These data indicate that the redox cycling steps of melanization are halted due to the inability of dopaquinone to be reduced into L-DOPA. This could also indicate that while L-DOPA was oxidized more in the presence of glyphosate, it may form a non-dopaquinone product-either a radical-mediated dimer with itself or a semiquinone.

\section{Discussion}

We investigated the effect of glyphosate on melanin production in 2 species of insects, G. mellonella and A. gambiae, and found that both glyphosate and its major metabolite AMPA were inhibitors of insect phenoloxidase and melanization. Although glyphosate and AMPA are relatively weak inhibitors of these insects' melanization, the inhibitory concentrations are relevant in the environment given the vast amounts used in agriculture, their environmental stabilities, and the high potential for insect-herbicide interactions. Therefore, glyphosate has a high potential to influence key insect physiological systems. We observed that glyphosate enhanced the susceptibility to infection of 2 phylogenetically distinct insects, G. mellonella and A. gambiae. This raises concerns and the suggestion that glyphosate may interfere broadly with insect 
immunity through its effects on melanin-based defenses. Analysis of in vitro tyrosinase and auto-oxidation models revealed that glyphosate inhibited melanization by acting as a synergistic antioxidant and disrupting redox cycling. Overall, our findings provide new insights on the complex reaction and suggest potential harmful effects of this herbicide on nontarget organisms, including some insects that may be important to ecosystem stability, and already in peril due to the threat of an "insect apocalypse."

G. mellonella treated with glyphosate were more susceptible to infection with C. neoformans. Glyphosate treatment was associated with reduced size of melanized nodules in the hemolymph following infection with C. neoformans. Two of three replicates showed significantly reduced numbers of melanized nodules in the glyphosate-treated infections. Nodules are primarily composed of hemocyte aggregates, released immune factors, and melanin encapsulation of the pathogen, which function together to kill invading pathogens [22]. Altogether, these data suggest that glyphosate weakened the melanin-based immune response of G. mellonella, which could have grave implications for host defense. Galleria are members of the order Lepidoptera (moths and butterflies), which represent up 10\% of known species on Earth. Interactions with glyphosate in the soil, on plants during pollination, or ingested through herbivory could contribute to immunocompromised lepidopteran populations. Glyphosate's effects on immunity in insects could compound a controversial and preexisting problem of declines in Lepidopteran biomass in recent decades [25,105-108].

Like our observations with G. mellonella, glyphosate made the A. gambiae mosquito more susceptible to $P$. falciparum parasite infection, the primary agent of human malaria in Africa. However, melanization is not considered the primary anti-P. falciparum immune response in this malaria model [109]. The increased susceptibility of A. gambiae to P. falciparum could be due to broader alterations of mosquito immune defenses, or disruption of non-melanin roles of catecholamine oxidation and phenoloxidase in insect immunity including the production of ROS, cytotoxic intermediates, and pathogen lysis $[3,13,110]$. Importantly, we observed that even when infections of A. gambiae with $P$. falciparum resulted in an overall low to no parasite burden in control-treated groups, glyphosate-treated groups exhibited a higher infection burden and prevalence. This is notable because Plasmodium oocyte development within the mosquito is a major bottleneck to successful vector competence in nature [111]. If a mosquito can prevent oocyst formation, there is no transmission of malaria to humans. The numbers of oocysts from these low parasite burden experiments are in line with the normal burdens found in natural field infection models $[112,113]$. Our data may indicate that mosquitoes exposed to glyphosate were less able to control Plasmodium infection they would have otherwise resisted, thereby becoming potentially better vectors for malaria. Overall, our results raise concerns for public health and malaria control initiatives in regions in which malaria is endemic and where there is increasing use of glyphosate, including areas of Latin America, sub-Saharan Africa, and Asia.

Our data revealed that uninfected adult female mosquitoes treated with glyphosate displayed a hormesis-like dose-dependent effect when measuring survival outcomes. Survival increased at low doses of glyphosate compared to the control. This greater longevity may be due in part to reduced basal damage from host defense mechanisms that normally occur during melanin formation, and/or altered gut microbiota. In contrast, mosquitoes exposed to high concentrations of glyphosate showed decreased survival. These data suggest the broader notion that glyphosate could have varied and complex outcomes on vector competence depending on its concentration in the environment. The low-concentration glyphosate treatments resulted in longer-lived, yet immunosuppressed, mosquitoes that were slightly more susceptible to infection with P. falciparum, whereas short-lived high glyphosate-treated mosquitoes were much more susceptible to $P$. falciparum. Further, while the $10 \mathrm{mM}$-treated mosquitoes had the 
worst survival outcome, the mosquitoes that survived the drugging showed low susceptibility to $P$. falciparum infection. These observations suggest a potentially interesting effect whereby very high concentrations of glyphosate reduce mosquito survival, but bolster the immune system or general physiology of survivors, which then allows them to resist $P$. falciparum infection with greater success. Alternatively, very high glyphosate treatment could be selecting for mosquitoes within the population more resistant to $P$. falciparum infection.

Our analyses of A. gambiae midgut microbiota indicated that glyphosate $\operatorname{did} \operatorname{not}$ impact $A$. gambiae midgut culturable bacterial density, although the herbicide did perturb midgut microbiota composition in a non-dose-dependent manner. More specifically, glyphosate altered diversity of the microbial community, and glyphosate-treated mosquitoes exhibited diminished Enterobacteriaceae and expanded Asaia spp. populations. The presence of some Enterobacteriacae, including the common insectary contaminant Serratia marcescens, in Anopheles spp. midguts is associated with lower susceptibility to Plasmodium spp. infection [114,115]. This effect is observed quantitatively by the significantly different prevalence of individual bacterial taxons (beta diversity) between the glyphosate and control-treated microbiota, while there is an overall unchanged number of bacterial taxa present (alpha diversity). Beta diversity analysis indicates that microbial communities associated with glyphosate-treated mosquitoes cluster together and are different than those from control mosquito communities.

Our results are consistent with reports that glyphosate perturbs the microbiota of honeybees that makes them more susceptible to infection [59]. Our data suggest that while glyphosate may perturb the microbiota and affect immunity as previously described [59,60], it can also inhibit melanization which is a critical part of insect immune defense. We do not see a dose-dependent effect of glyphosate on the microbiota composition, but we do see a dosedependent effect on mosquito susceptibility to Plasmodium infection; this indicates that the enhanced susceptibility might be unrelated to microbiome perturbations. These mechanisms of susceptibility are not mutually exclusive and could be additive to weaken insect health. Additionally, while AMPA does not disrupt the microbiota of honeybees [60], we show it can inhibit melanization of G. mellonella phenoloxidase and mushroom tyrosinase. A recent study in Apis cerana cerana honeybees indicate that glyphosate-based herbicide treatment increases expression of wound and defense genes, including those related to melanization [116]. Interestingly, this study also showed that glyphosate feeding decreased the expression of many odorant binding proteins, which have been shown to mediate the melanization response in both tsetse fly (Glossina morsitans morsitans) and Drosophila melanogaster [117] This suggests complex regulation of melanization following treatment with glyphosate-based herbicide, including the possibility of increased melanin-related gene expression as a compensation for glyphosate's inhibitory effects. Additionally, other surfactants and components of the commercial herbicide formulation used could trigger damage and immune gene expression.

Melanins and phenoloxidases are involved in other physiological functions in insects including proper pupation and cuticle and eggshell development. In our experiments, which involved single dosing or short duration of feeding, we did not detect a difference in coloration of adult mosquito treated with glyphosate, nor a defect in G. mellonella pupation following glyphosate treatments of final instar larvae. However, we cannot rule out that longer glyphosate exposure or feeding throughout the lifecycle would affect these functions. If such effects happen, they will only compound the effects of glyphosate on melanin-based immunity and insect physiology.

We sought to understand the mechanism of melanization inhibition by glyphosate. The process of melanization is highly dependent upon oxidation and redox cycling between catechols and quinones. The melanin production process is halted if the oxidizing ability and the redox potentials are altered. Melanization begins with the conversion of L-DOPA into 
dopaquinone through enzymatic or spontaneous oxidation of L-DOPA, followed by redox cycling that results in dopachrome formation and, subsequently, melanin polymerization. Glyphosate inhibited formation of dopaquinone and melanin pigment mediated by both tyrosinase and auto-oxidation, which strongly suggests that glyphosate inhibits L-DOPA oxidation in an enzyme-independent manner. We found that other phosphate-containing compounds inhibited melanization in a similar manner including phosphoserine, phosphoacetic acid, pyrophosphate, and phosphoric acid. This is in line with literature reports that other aminophosphonic acids inhibit fungal eumelanin in the human pathogen Aspergilius flavus [118]. Incidentally, this class of compounds is patented for use in human cosmetics and is marketed as solutions to inhibit melanogenesis in the skin $[119,120]$.

We found no evidence that glyphosate irreversibly inhibited tyrosinase activity or directly interfered with enzyme function. Addition of copper ions did not rescue the inhibition, indicating that the copper-based catalytic core of tyrosinase is not disrupted by glyphosate. Interestingly, low copper increased tyrosinase activity and high doses reduced activity. However, copper had minimal effects on tyrosinase activity during high glyphosate concentrations. It appears that glyphosate, possibly through chelation, acts as a "buffer" of copper ions and can reduce the metal's harmful effects, similar to previous findings concerning the toxicity of highcopper soil to earthworms following glyphosate treatment [96]. This could have broader implications for melanogenesis in nature, where some fungi use copper as a signal to up-regulate melanin-producing enzymes [121], and thus copper ion sequestration could reduce melanin production.

We examined the ability of glyphosate and the other compounds to quench free radicals, which are necessary to the melanization process. Of the inhibitors tested, only glyphosate had radical-quenching activity, but this occurred relatively slowly compared to the typical timeframe of antioxidant reactions reported in literature [122]. This property is likely not the mechanism of inhibition as phosphoserine has a similar structure and near identical inhibition of melanization as glyphosate, yet no radical-quenching properties. While not a free-radical quencher, phosphoric acid is a known antioxidant synergist-a class of compounds that enhance antioxidant properties of phenolic compounds by chelating metals or reverting antioxidants into their active states [99]. Synergists like phosphoric acid, citric acid, malic acid, and alpha-hydroxy acids are added to foods, medicines, and cosmetics at concentrations up to $10 \%$ as a preservative due to their synergist effects on antioxidants [123]. Glyphosate behaved similarly to phosphoric acid and citric acid; citric acid inhibited melanization similarly to glyphosate and phosphoric acid, suggesting an inhibition mechanism via antioxidant synergy. Additionally, we report that glyphosate and other inhibitors have synergistic effects on the antioxidant properties of L-DOPA. L-DOPA's antioxidant properties derive from its reduction back to a normal state from an oxidized state, or a radical-mediated adduction reaction with the oxidized compounds in solution. Since glyphosate makes L-DOPA a more efficient antioxidant, glyphosate thus alters the oxidative balance of L-DOPA and/or produces a buildup of radical or semiquinone intermediates.

Consistent with these findings of antioxidant synergy, cyclic voltammetry revealed that glyphosate decreased the L-DOPA-Dopaquinone redox potential. Hence, L-DOPA becomes both a weaker oxidizing agent and a stronger reducing agent (antioxidant) and is more prone to oxidation in the presence of the herbicide. Glyphosate decreased dopaquinone reduction in a dose-dependent fashion indicating that dopaquinone cannot be reduced or is not produced following L-DOPA oxidation. A lack of dopaquinone could indicate that glyphosate causes oxidized L-DOPA semiquinone intermediates to remain stable or react with each other and form L-DOPA dimers. On the other hand, if dopaquinone cannot be reduced into L-DOPA, melaninization becomes unfavorable as redox exchange could not occur. These changes in 
voltammogram do not appear when the L-DOPA solution is treated with $16 \mathrm{mM}$ glycine but did occur with citric acid. This further supports that glyphosate is acting as a synergistic antioxidant and prevents the redox-dependent melaninization.

Our findings investigating glyphosate's mechanism of melanin inhibition points to disruption of oxidative balance and redox cycling which may result in the buildup of toxic oxidative intermediates. Previous studies evaluating glyphosate's impact on organisms show that the herbicide increases oxidative stress, lipid peroxidation, and antioxidant responses in bacteria, plants, arthropods, fish, amphibians, rats, and human red blood cells [69-76]. These data bolster our findings that glyphosate promotes oxidation in phenolic compounds like L-DOPA and inhibits clearance of oxidative stress. Understanding the mechanisms by which compounds such as glyphosate might impact insect biomass and contribute to a potential insect decline is important, as they have both direct and indirect impacts on human health.

Glyphosate's interference with melanization could have considerable environmental impact given its stability and wide concentration range, from over $50 \mathrm{mM}$ at time and at site of application to under $1 \mathrm{nM}$ in runoffs from application sites $[33,39,124]$. At higher concentrations, glyphosate could inhibit melanin production in some insects, thus rendering them more susceptible to pathogens due to reduced immune competence. This suggests protean consequences for human health ranging from ecosystem disruption to altered vector competency of lethal human pathogens and increased malaria transmission in endemic regions that use glyphosate-based herbicides in agriculture. Importantly, we provide evidence that glyphosate enhances A. gambiae susceptibility to the human malaria parasite, which could potentially make it a better vector for transmitting disease to humans. Our data in Galleria and Anopheles can perhaps be extrapolated to other lepidopteran (moth and butterfly) and dipteran (fly) species with additional importance to the environment.

In summary, our results suggest that glyphosate interferes with melanization in 2 insect species, through a mechanism involving altering the redox potential of melanin polymerization reaction. This phenomenon is concerning because of the importance of melanization in insect immunity. A strong immune response is vital for insect survival, and disruption of their immune function, including the inhibition of melanization, could be disastrous for these animals. Insects are pivotal members of the world's ecosystems, essential to maintaining proper function, and they ensure human food security. Yet, certain data indicate a drop in insect biomass over recent decades, a phenomenon that has been called the "insect apocalypse" $[25,105,106,125,126]$. Although this view has been questioned regarding the true extent and possible causes of the insect population declines [127-131], our results suggest that glyphosate use as a mechanism by which insect immunity can be undermined by human activities.

\section{Methods}

\section{Biological materials}

G. mellonella larvae were obtained through Vanderhorst Wholesale, St. Marys, Ohio, USA. C. neoformans strain $\mathrm{H} 99$ (serotype A) was kept frozen in $20 \%$ glycerol stocks and subcultured into Sabouraud dextrose broth for 48 hours at $30^{\circ} \mathrm{C}$ prior to each experiment. The yeast cells were washed twice with PBS, counted using a hemocytometer (Corning, New York, USA), and adjusted to $10^{6}$ cells $/ \mathrm{ml}$.

A. gambiae (Keele strain) mosquitoes were maintained on sugar solution at $27^{\circ} \mathrm{C}$ and $70 \%$ humidity with a 12-hour light to dark cycle according to standard rearing condition. P. falciparum NF54 (Walter Reed National Military Medical Center, Bethesda) infectious gametocyte cultures were provided by the Johns Hopkins Malaria Research Institute Parasite Core Facility 
and were diluted to $0.05 \%$ gametocytemia with naïve human blood before feeding to the mosquitoes using an artificial glass membrane feeder as established in [84].

\section{Compound and dilution preparation}

Each compound, including the glyphosate (Millipore Sigma, Product \#45521), was prepared in $300 \mathrm{mM}$ stock solution in Milli-water Q and brought to a pH of 5.5, and $20 \mu \mathrm{l}$ of each compound was serially diluted 1:2 in PBS (pH 7.4), with a compound-free control. When all reaction components are added, the final concentrations of the compounds in these dilutions were $33.33,16.67,8.33,4.17,2.08,1.04,0.52$, and $0 \mathrm{mM}$.

\section{Galleria mellonella hemolymph extraction and phenol oxidase activity}

Healthy (active and cream-colored) larvae were cold anesthetized and punctured in their proleg with $18 \mathrm{G}$ needle, and pressure was applied to the larvae to promote bleeding of hemolymph. Hemolymph was collected from larvae directly into an eppendorf tube. Anticoagulants were not used as they might interfere with the melanization process.

For automelanization experiments, hemolymph was diluted 1:10 in PBS and mixed with a pipette. Then, $160 \mu \mathrm{l}$ of 1:10 hemolymph is added to $20 \mu \mathrm{l}$ of glyphosate serially diluted in PBS. The change in absorbance at $490 \mathrm{~nm}$ was recorded and analyzed as described above.

For experiments with L-DOPA, hemolymph was diluted 1:5 in PBS and mixed by pipette. Experiments were performed as per the phenoloxidase activity assay in [132].

To test the effect of glyphosate on hemocytes viability, hemolymph was diluted 1:2 with anticoagulation buffer [133], as melanization was not of importance for this experiment. Hemocytes were pelleted and suspended in anticoagulation buffer. Glyphosate was added to an aliquot of hemocytes in solution and incubated with mixing on a rocker at $30^{\circ} \mathrm{C}$ for $15 \mathrm{~min}$ utes. Hemocyte viability was assessed by $0.02 \%$ trypan blue staining and enumeration of stained (dead) versus unstained (alive) hemocytes with a hemocytometer.

\section{Galleria mellonella infection and survival}

Healthy final instar G. mellonella larvae weighing between 175 and $225 \mathrm{mg}$ were selected and left starving overnight. Groups of larvae were injected with $10 \mu \mathrm{l}$ of PBS or $10 \mu \mathrm{l}$ of $1 \mathrm{mM}$ sterile glyphosate in PBS. Larvae were monitored and left to recover for 5 hours. Larvae were then injected with $10 \mu \mathrm{l}$ of sterile PBS or injected with $10^{4} \mathrm{C}$. neoformans yeast cells per larva. Due to the low concentration of glyphosate administered to the larvae, their volume of hemolymph, and their body volume, we believe the approximate concentration of glyphosate is below the concentrations required to inhibit C. neoformans growth [26]. G. mellonella larvae and pupae were kept at $30^{\circ} \mathrm{C}$ and monitored daily for survival for 14 days. Survival was assessed by movement upon stimulus with a pipette. See S9A Fig.

\section{Melanization and nodule measurements}

G. mellonella larvae were drugged and infected as described above in groups of 3 larvae per condition. After 24 hours, larval hemolymph was removed directly in anticoagulation buffer, centrifuged at 10,000 $\mathrm{xg}$ for 5 minutes, and resuspended in coagulation buffer.

Brightfield microscopy images were randomly taken at $4 \times$ magnification, with 15 to 20 images taken per condition per replicate. These images were analyzed using Fiji [134] Particle Analyzer function using with a threshold set between 0 and 120 mean gray value. Particle area and numbers were calculated. Additional images were taken of nodules at $20 \times$ and $100 \times$ magnification, the latter of which were used to manually score the degree of melanization of fungal 
cells within the nodules. Statistical significance of differences between melanized particle area was analyzed using a nested nonparametric Mann-Whitney-Wilcoxon rank test using the nestedRanksTest package (Version 0.2, D.G. Scofield, 2014) [77] in R for R 4.0.2 GUI 1.72 for Mac OS at https://www.r-project.org/ (R Core Team, 2020).

\section{Anopheles gambiae phenoloxidase activity}

Phenoloxidase activity assays were performed as previously described [132]. Experiments were done in biological triplicate with different batches of mosquitoes, as well as in technical triplicate per biological replicate of 3 batches of 10 mosquitoes.

\section{Anopheles gambiae survival}

Adult female mosquitoes of A. gambiae Keele strain were raised on 10\% sucrose for 3 days postemergence. On the third day, adult females were sorted into 7 groups of 40 and placed into mesh-covered cardboard cups and provided a cotton ball with $10 \%$ sucrose mixture with either $0 \mu \mathrm{M}$ (Control), $30 \mu \mathrm{M}, 100 \mu \mathrm{M}, 300 \mu \mathrm{M}, 1 \mathrm{mM}, 3 \mathrm{mM}$, or $10 \mathrm{mM}$ glyphosate. The cotton balls were replaced every third day with new cotton balls and fresh sucrose/glyphosate solutions. Mosquito death was monitored daily for 14 days. Experiments were performed in 3 independent replicates, for a total of 120 mosquitoes in each treatment group.

\section{Anopheles gambiae cuticle pigmentation and wing size}

Adult female mosquitoes were drugged for 5 days as previously described. Mosquitoes were cold euthanized and mounted dorsally on a slide with double-sided tape. Images of the mosquito ventral abdomen were taken under a dissection microscope with constant exposure and lighting conditions. Pigmentation was measured using Fiji software [134]. The entire abdomen of each mosquito was selected using a freehand selection tool, and the 8-bit mean gray value was measured using the Measure tool. A measurement of 0 corresponds to a pure black gray value, whereas 255 corresponds to a pure white gray value.

Following abdomen pigmentation measurements, mosquito bodies were removed, with careful attention to keeping the wings remaining intact on the tape. Intact wings were imaged on a microscope, and the length of the individual wing lengths were measured from tip to tip using Fiji Measure tool.

\section{Anopheles gambiae infection with Plasmodium falciparum}

Adult female mosquitoes ( 3 to 4 days old) of A. gambiae Keele strain were sorted and drugged as described above. On the fifth day of glyphosate exposure, mosquitoes were provided a blood meal containing P. falciparum. Blood-fed engorged mosquitoes were sorted on ice and fed $10 \%$ sucrose ad libitum for 8 days. Midguts were dissected and stained with $0.2 \%$ Mercurochrome solution, and oocysts were enumerated using a 20X objective with light microscopy. See S9B Fig.

\section{Anopheles gambiae midgut microbiome analysis}

Adult female mosquitoes ( 3 to 4 days old) of A. gambiae Keele strain were sorted and drugged as described above. On the fifth day of glyphosate exposure, mosquitoes were sterilized in ethanol for 2 minutes, washed, and dissected in sterile PBS. The midguts were removed, placed in $500 \mu \mathrm{l}$ sterile PBS on ice, homogenized, diluted, and plated on LB agar plates. Plates were incubated at $30^{\circ} \mathrm{C}$ for 3 days, and individual colonies were counted. Each experiment used 10 to 20 mosquitoes per condition, and the experiment was performed 3 independent times. 
For the 16S rRNA sequencing studies, mosquitoes were reared and drugged, and then midguts were dissected as described above, with 5 individual midguts per condition. DNA was extracted from frozen mosquito samples using the Lucigen EpiCentre MasterPure DNA extraction kit (Lucigen, Middleton, Wisconsin, USA). The bacterial 16S rRNA gene was amplified by PCR, and sample-specific Illumina adapters were ligated to the PCR products. PCR products from multiple samples were pooled and sequenced on the Illumina MiSeq platform by the University of Connecticut MARS Facility. Data were then analyzed using mothur [135] to construct contigs to align forward and reverse reads, remove ambiguous bases and chimeric regions, align sequences to the Silva $16 \mathrm{~S}$ V4 reference database, and cluster reads into 3\% operational taxonomic units (OTUs). Sequences derived from known contaminants were selectively removed. Alpha and beta diversity measurements were performed using the Shannon diversity index and Bray-Curtis dissimilarity distance, respectively. Bray-Curtis distances were graphed on principal coordinates analysis (PCoA) plots in 2 dimensions. Taxa and PCoA graphs were produced using MicrobiomeAnalyst [136,137]. See S9C Fig.

\section{Dopaquinone formation MBTH assay}

Quinones like dopaquinone are unstable and difficult to study directly; thus, dopaquinone quantification relies on the formation of a stable adduct with MBTH (3-methyl-2-benxothiaxolinone hydrazine) that forms a pigment that absorbs at $505 \mathrm{~nm}$ [138]. This absorption overlaps with the absorption of another melanin intermediate, dopachrome $(\mathrm{Q})$, but is not expected to interfere since dopaquinone reaction with MBTH prevents dopachrome formation. Further, the molar absorbance coefficient for MBTH-Dopaquinone is more than 10 times higher $(39,000 \mathrm{~L} /[\mathrm{mol} \mathrm{cm}])$ than that of dopachrome $(3,700 \mathrm{~L} /[\mathrm{mol} \mathrm{cm}])$, and interference from dopachrome would be relatively small.

MBTH reaction mixtures were prepared as previously described [138]. This mixture is warmed at $42^{\circ} \mathrm{C}$ to help solubilize the components. Then, $5 \mu \mathrm{lof} 2 \mu \mathrm{g} / \mathrm{ml}$ mushroom tyrosinase (Sigma, Product \#T382) and $20 \mu \mathrm{l}$ of $20 \mathrm{mM} \mathrm{L-DOPA}$ are added to the MBTH solution, and $160 \mu \mathrm{l}$ of the solution is immediately added to each well containing compounds. The plate was read at an absorbance of $505 \mathrm{~nm}$ for 30 minutes at $30^{\circ} \mathrm{C}$ and read again at 1 hour and overnight. The dopaquinone levels are determined by the formation of the bright pink adduct between the quinone and the MBTH.

\section{Dopachrome and melanin measurements}

Tyrosinase activity was determined as previously described [132], substituting mushroom tyrosinase for phenoloxidase. The formation rate of dopachrome is measured as the maximum velocity of this reaction, and the dopachrome levels are measured as the absorbance at $490 \mathrm{~nm}$ after 30 minutes as the absorbance values plateau. Melanin levels are measured as the absorbance at $490 \mathrm{~nm}$ after the reaction has continued for 5 days in the dark at room temperature.

\section{Free-radical scavenging ABTS assay}

ABTS solution was prepared as previously described [139]. To test the radical-scavenging capability of the compounds, $10 \mu \mathrm{l}$ of the compounds were serially diluted in a 96-well plate as previously described, and $90 \mu \mathrm{l}$ of diluted ABTS was added to each well. The $734 \mathrm{~nm}$ absorbance was measured immediately, after 10 minutes, 1 , and 2 hours. In kinetics experiments, absorbance readings were taken every 2 minutes for 5 hours.

To measure the radical scavenging capacity of the synergistic compounds and L-DOPA mixtures, ABTS was prepared and diluted in Milli-Q water. In each well, $5 \mu$ of compound stocks were added with either $5 \mu \mathrm{l}$ of water or $5 \mu \mathrm{l}$ of $500 \mu \mathrm{M}$ L-DOPA. Next, $90 \mu \mathrm{l}$ of ABTS 
solution was added to the well, and the absorbance was read immediately at $734 \mathrm{~nm}$. Synergy was calculated from these data using the following formula:

$$
\text { Synergy Ratio }=\frac{(\Delta \text { Abs } 734 \text { Compound Alone }+\Delta \text { Abs } 734 \text { DOPA Alone })}{\Delta \text { Abs } 734 \text { Compound with DOPA }}
$$

\section{Glyphosate effect on L-DOPA}

To determine if L-DOPA is reacting with glyphosate, we analyzed by NMR. We diluted 300 $\mathrm{mM}$ stock of glyphosate in water to $60 \mathrm{mM}(10 \mathrm{mg} / \mathrm{ml})$ in $\mathrm{D}_{2} \mathrm{O}$, prepared $20 \mathrm{mM}(4 \mathrm{mg} / \mathrm{ml})$ L-DOPA in $\mathrm{D}_{2} \mathrm{O}$, and prepared 2 mixtures of glyphosate and L-DOPA: one with $20 \mathrm{mM}$ (4 $\mathrm{mg} / \mathrm{ml}) \mathrm{L}-\mathrm{DOPA}$ and $60 \mathrm{mM}(10 \mathrm{mg} / \mathrm{ml})$ of glyphosate in $\mathrm{D}_{2} \mathrm{O}$, and another with a low concentration of $1 \mathrm{mg} / \mathrm{ml}$ for both compounds equaling $5 \mathrm{mM} \mathrm{L-DOPA}$ and $6 \mathrm{mM}$ glyphosate. We then performed ${ }^{31} \mathrm{P}-\mathrm{NMR}$ and ${ }^{1} \mathrm{H}-\mathrm{NMR}$ on these samples.

\section{Glyphosate effect on tyrosinase}

To determine the tyrosinase kinetics with glyphosate as an inhibitor, we serially diluted $155 \mu \mathrm{l}$ of $20 \mathrm{mM}$ L-DOPA in Milli-Q water. To each dilution of L-DOPA, we added $20 \mu \mathrm{l}$ of glyphosate diluted in PBS and $5 \mu \mathrm{l}$ of $2 \mu \mathrm{g} / \mathrm{ml}$ mushroom tyrosinase to the reaction mix. In order to account for nonenzymatic oxidation of L-DOPA, we ran an experiment in parallel, in which we added $5 \mu$ of Milli-Q water instead of tyrosinase. The reaction mix was kept at $30^{\circ} \mathrm{C}$ for 24 hours. The plate was read at $490 \mathrm{~nm}$. To calculate enzyme-specific oxidation of L-DOPA, the no enzyme values were subtracted from the tyrosinase rows. The kinetics curve is plotted as a function of absorbance after 24 hours of reaction time versus concentration of L-DOPA.

We tested if tyrosinase concentration had an effect on the percent inhibition of the reaction. We prepared dilutions of tyrosinase. We added $5 \mu \mathrm{l}$ of each dilution to a 96-well plate and added $135 \mu \mathrm{l}$ of Milli-Q water, $20 \mu \mathrm{l}$ of $20 \mathrm{mM} \mathrm{L-DOPA}$, and $20 \mu \mathrm{l}$ of glyphosate in PBS. We measured maximum velocity of this reaction at $490 \mathrm{~nm}$. The difference in velocities and percent inhibition reported were calculated by difference $=V_{\max }$ water $-\mathrm{V}_{\max \text { glyph }}$, and percent inhibition $=100^{*}\left(\mathrm{~V}_{\max \text { glyph }} / \mathrm{V}_{\max \text { water }}\right)$.

To determine if glyphosate irreversibly affects tyrosinase activity, $450 \mu \mathrm{lof} 20 \mu \mathrm{g} / \mathrm{ml}$ mushroom tyrosinase was prepared in $450 \mu \mathrm{l}$ of $50 \mathrm{mM}$ sodium phosphate buffer ( $\mathrm{pH} 7$ ), either with $50 \mu \mathrm{l}$ of $300 \mathrm{mM}$ glyphosate or $50 \mu \mathrm{l}$ of Milli-Q water. The enzyme solution was loaded into a hydrated 10,000 MWCO Slide-a-lyzer dialysis cassette (Thermo Scientific, Waltham, Massachusetts, USA), and the enzyme solutions were dialyzed in a 50-mM sodium phosphate buffer at $4^{\circ} \mathrm{C}$, according to the manufacturer's protocol. Protein concentrations were measured and normalized using sodium phosphate buffer. To measure the kinetics of the control enzyme versus the treated enzyme, a kinetics assay was performed as previously described. Each reaction's maximum velocity is determined and plotted.

\section{Copper rescue of melanin inhibition}

As previously described, serial dilutions of glyphosate were arrayed in 8 rows; 1 row per copper ion concentrations to be tested. Copper sulfate was prepared and serially diluted, and $10 \mu \mathrm{L}$ of the copper solution is added to each well containing the glyphosate dilution. To each well, $150 \mu \mathrm{L}$ of reaction mix ( $125 \mu \mathrm{L}$ of Milli-Q water, $20 \mu \mathrm{L}$ of $20 \mathrm{mM} \mathrm{L}-\mathrm{DOPA}$, and $5 \mu \mathrm{L}$ of $2 \mu \mathrm{g}$ / $\mathrm{mL}$ mushroom tyrosinase ( $5 \mu \mathrm{l}$ of water used for auto-oxidation experiments) was added. The final copper ion concentrations were $400,200,100,50,25,12.5,6.25$, and $0 \mu \mathrm{M}$. The dopachrome and melanin measurements are reported as previously described. 


\section{Cyclic voltammetry}

Cyclic voltammetry was performed using a Metrohm Autolab potentiostat (Switzerland), 3 $\mathrm{mm}$ Glassy Carbon working electrode, $10 \mathrm{~mm} \times 10 \mathrm{~mm} \times 0.1 \mathrm{~mm}$ platinum plate counter electrode, and an $\mathrm{Ag} / \mathrm{AgCl}$ reference electrode in $3 \mathrm{M} \mathrm{KCl}$ solution. Solutions were prepared in 0.1x PBS (Difco, Franklin Lakes, New Jersey, USA) at a pH 6.00, adjusted with $\mathrm{NaOH}$ and $\mathrm{HCl}$. A volume of $10 \mathrm{~mL}$ of L-DOPA solution was freshly prepared in this buffer, and $1 \mathrm{~mL}$ of glyphosate, glycine, water, etc., solution at $\mathrm{pH} 6.00$ were added to the L-DOPA. Readings were done with 3 tracings at a scan rate of $50 \mathrm{mV} / \mathrm{s}$ at intervals of $5 \mathrm{mV}$ steps. Glassy carbon electrode was washed and polished between readings with slurry of alumina powder and water on cloth pads.

\section{Supporting information}

S1 Fig. G. mellonella supplemental data. (A) Broad-spectrum protease inhibitor (cOmplete, Roche, Basel, Switzerland) was added to G. mellonella hemolymph to prevent the activation of new phenoloxidase and to control for any impact that glyphosate may have on phenoloxidase activation cascade, cell viability, and gene expression. The general trend remains the same that glyphosate inhibits phenoloxidase activity with and without protease inhibitor, albeit lower with protease inhibitor due to the lower concentration of activated enzyme. (B) Phenoloxidase activity was assessed using exogenous L-DOPA for 1 batch of G. mellonella, during these experiments, the lower concentration of glyphosate resulted in increased phenoloxidase activity as compared to the control. This suggests that there may be some cellular regulation of phenoloxidase induced by glyphosate. It is possible that the doses of glyphosate tested elicit some cellular response that increases phenoloxidase expression, secretion, and/or activation as a feedback/hormesis-like response to the reduced melanin production. These data represent 3 independent replicates, but this pattern of enzymatic activity as a function of glyphosate concentration was not seen in subsequent batches of larvae. (C) Hemocyte viability was not dramatically affected by concentrations of glyphosate ranging from $100 \mu \mathrm{M}$ to $10 \mathrm{mM}$, indicating that our data are likely not artifacts of cytotoxic concentrations of glyphosate. Error bars in (A-C) represent $\pm S D$. (D) AMPA, a major metabolite of glyphosate, inhibits tyrosinase-mediated melanization similar to glyphosate. Grayscale bars represent mean absorbance at $490 \mathrm{~nm}$ relative to no compound control. The darker colors correspond to increased pigment formation. (E) Larvae treated with glyphosate and subsequently infected with lac1s mutant C. neoformans strain showed a similar pattern of increased susceptibility as the wild-type H99, although the differences in susceptibility with the lac1 $\Delta$ infected larvae are not statistically significant. Each experiment represents at least 3 independent replicates. The PBS mock infection condition represents survival of 95 animals, over the span of 4 biological replicates, and 6 total technical replicates. The lac1 14 mutant infection represents survival of 75 animals over the span of 4 biological replicates. The PBS mock infection data are the same as the data in Fig 5B, as all the infections were done concurrently under the same conditions. (F) Single injection of $10 \mu \mathrm{l}$ of $1 \mathrm{mM}$ glyphosate does not affect the pupation of G. mellonella at $30^{\circ} \mathrm{C}$ and RT. Data from $30^{\circ} \mathrm{C}$ represent $25-35$ animals for each group over 2 biological replicates, and data from RT represent 45 animals from each group over 3 biological replicates. Statistical analysis performed using log-rank Mantel-Cox tests. (G-I) The 3 individual replicates from Fig 1D showing the size of the dark melanized particles within nodules are significantly smaller in the glyphosate-treated infected groups compared to the PBS-treated infected groups, with (G) and (H) showing that there were more melanized spots in the PBS-treated infected group compared to the glyphosate-treated. All statistical analyses performed using GraphPad Prism version 8.4.3 for Mac OS, GraphPad Software, San Diego, California, USA, www.graphpad.com. 
For underlying data, please see Data Availability section and/or S1 Table. AMPA, aminomethylphosphonic acid; Glyph, glyphosate; L-DOPA, 3,4-dihydroxyphenylalanine; RT, room temperature.

(TIFF)

S2 Fig. Low efficiency Plasmodium falciparum infection of A. gambiae and effects on mosquito cuticle. (A). Oocyst count per midgut for mosquitoes treated with or without glyphosate and infected with high-passage P. falciparum gametocyte culture, resulting in a low efficiency infection. Data represent 1 biological replicate. Dotted black line indicates $y=0$. Black lines for each condition indicate median oocyst count per midgut. We have chosen not to include the data from this replicate in the data shown in Fig 6, because the results from this one-off replicate appear due to poorly infectious parasite culture. Additionally, it is difficult to make comparisons using the low infection burden of the control group with the treatment groups, as well other replicates with higher oocyst burdens. (B) Infection prevalence (percent midguts with at least 1 oocyst) from the experiment described in (A). Fisher exact test performed for each condition individually compared to control and corrected for multiple comparisons using the Bonferroni method. (C) A total of 5 days of $1 \mathrm{mM}$ glyphosate treatment in adult female mosquitoes does not influence the abdomen's cuticular darkness as measured by mean gray value with 0 being pure black and 255 being pure white. Data representative of 2 biological replicates with 88 mosquitoes measured per condition. (D) Wing length, as a proxy for body mass and size, is not affected by 5 days of glyphosate treatment. Data representative of a single biological replicates with 32-36 mosquitoes measured per condition. Line and error bar represent mean $\pm \mathrm{SD}$ in $(\mathbf{C}, \mathbf{D})$. Unpaired $t$ test performed to determine statistical significance in (C, D). All statistical analyses performed using GraphPad Prism version 8.4.3 for Mac OS, GraphPad Software, San Diego, California, USA, www.graphpad.com. For underlying data, please see Data Availability section and/or S1 Table. Glyph, glyphosate; n.s., not significant. (TIFF)

S3 Fig. Glyphosate affects the A. gambiae microbiota in a dose-independent manner. (A) At the class level, glyphosate leads to an enrichment of Alphaproteobacteria and a depletion in Gammaproteobacteria. Tables showing the relative abundance of bacterial classes (B) and individual bacterial genera (C) following glyphosate treatment. (D) Alpha diversity does not follow a distinctive pattern with increasing glyphosate dose. (E) Glyphosate-treated and control-treated microbiota cluster separately in ordination space, but the clusters are not dose dependent. For underlying data, please see Data Availability section and/or S1 Table. (TIFF)

S4 Fig. Reaction of glyphosate with L-DOPA. Representative ${ }^{1} \mathrm{H}$ NMR spectra of $60 \mathrm{mM}$ glyphosate solution in $\mathrm{D}_{2} \mathrm{O}$ (Green), $20 \mathrm{mM}$ L-DOPA solution in $\mathrm{D}_{2} \mathrm{O}$ (Red), and $20 \mathrm{mM}$ L-DOPA mixed with $60 \mathrm{mM}$ glyphosate in $\mathrm{D}_{2} \mathrm{O}$ (Blue). There appears to be no shift in ${ }^{1} \mathrm{H}$ peaks and no appearance of new peaks, which is indicative of no reaction occurring between the compounds. Data representative of findings from 3 independent replicates. For underlying data, please see Data Availability section and/or S1 Table.

(TIFF)

S5 Fig. Glyphosate appears to "buffer" copper concentration in solution. High doses (2-16 $\mathrm{mM}$ ) of glyphosate prevent the enzymatic activity enhancing effects of lower copper concentration (6.25-25 $\mu \mathrm{M})$, but high doses of glyphosate also prevent the enzyme inhibitory effects of high copper concentration $(100-400 \mu \mathrm{M})$. Error bars represent \pm SD. Data represent 2 independent replicates. For underlying data, please see Data Availability section and/or S1 Table. (TIFF) 
S6 Fig. Antioxidant properties of glyphosate. (A) Change in absorbance of ABTS solution at $734 \mathrm{~nm}$ over time for $33.33 \mathrm{mM}$ glyphosate relative to the no glyphosate control. This indicates glyphosate quenches free radicals over an extended period of time. (B) Calculated antioxidant radical scavenging synergy between compounds tested and L-DOPA. Values represent the mean of at least 3 independent replicates. Error bars represent $\pm S D$. For underlying data, please see Data Availability section and/or S1 Table. ABTS, 2,2' -azino-bis(3-ethylbenzothiazoline-6-sulfonic acid); L-DOPA, 3,4-dihydroxyphenylalanine.

(TIFF)

S7 Fig. Glyphosate inhibits melanin production independent of L-DOPA concentration. (A) Inhibitory concentrations of glyphosate are not affected by L-DOPA concentration. This indicates that glyphosate is not reacting proportionately with L-DOPA as measured by absorbance at $490 \mathrm{~nm}$ after 5 days of reaction, relative to the no glyphosate control and with background absorbance subtracted. (B) The $\mathrm{IC}_{50}$ of glyphosate remains constant at approximately $1 \mathrm{mM}$ relative inhibition of melanin production appears dependent on glyphosate concentration alone and not on L-DOPA to glyphosate ratio. Data in panels (A) and (B) represent 2 alternative visualizations of the same experimental data. Error bars represent \pm SD. Each experiment represents at least 3 independent replicates. Grayscale bars represent mean absorbance at $490 \mathrm{~nm}$ relative to no compound control. The darker colors correspond to increased pigment formation. Red line represents the approximate $\mathrm{IC}_{50}$. Crossed out boxes represent values with no data. For underlying data, please see Data Availability section and/or S1 Table. L-DOPA, 3,4-dihydroxyphenylalanine. (TIFF)

S8 Fig. Cyclic voltammetry supplemental data. (A) Peak 1 was validated as the oxidation of L-DOPA, and Peak 2 was validated as the reduction peak of dopaquinone by correlating increased peak intensity with increasing concentration of L-DOPA under the same potentiostat parameters. (B) Glycine (16 mM) - a non-phosphate analog of glyphosate, a noninhibitor of melanization, and a nonantioxidant-does not alter the oxidation potential of L-DOPA. Conversely, citric acid (16 mM) - a known synergistic antioxidant and inhibitor of melanization-does alter the oxidation potential of L-DOPA in similar ways as glyphosate. The L-DOPA alone control and glyphosate voltammograms in panel (B) are the same as those found in Fig 7E. Each experiment represents at least 3 independent replicates, with 3 cycles per replicate. The tracings represent the mean value of the 3 replicates over the course of 3 cycles. For underlying data, please see Data Availability section and/or S1 Table. L-DOPA, 3,4-dihydroxyphenylalanine.

(TIFF)

S9 Fig. Experimental methods diagram for insect experiments. (A). During G. mellonella infection with C. neoformans, larvae were injected with $10 \mu \mathrm{l}$ of $1 \mathrm{mM}$ glyphosate, left to recover for 5 hours, and were subsequently infected with $10^{4}$ cells/larvae of C. neoformans H99 strain. Survival was monitored for 14 days. (B) During A. gambiae infection with P. falciparum, mosquitoes were drugged with glyphosate-laced $10 \%$ sucrose solution for 5 days, then fed with a P. falciparum-infected blood meal, and fed $10 \%$ sucrose for 8 days. On day 8 , mosquitoes were dissected, and the midguts were stained with mercurochrome to facilitate oocyst enumeration. (C) Glyphosate-drugged mosquitoes were dissected under sterile conditions, and 5 midguts were collected individually per condition. DNA was extracted from samples and bacterial $16 \mathrm{~S}$ rRNA genes were amplified by PCR and sample-specific Illumina adapters were ligated to products. PCR products were pooled and sequenced on the Illumina MiSeq 
platform. Data were then analyzed using mothur to construct contigs, align reads, remove ambiguous bases and chimeric regions, align sequences to the Silva 16S V4 reference database, and cluster reads into 3\% OTUs) Sequences from known contaminants were removed. Alpha and beta diversity measurements were performed using the Shannon diversity index and Bray-Curtis dissimilarity distance, respectively, and plotted using MicrobiomeAnalyst. Figures made with BioRender. OTU, operational taxonomic unit.

(TIFF)

S1 Table. Links to the underlying data for each figure in the Figshare data repository. (XLSX)

\section{Acknowledgments}

We would like to acknowledge Dr. Gene Fridman in the Johns Hopkins School of Medicine for lending the use of the Fridman lab's Metrohm Autolab potentiostat for use in the cyclic voltammetry experiments. We would like to thank the Johns Hopkins Malaria Research Institute and the Department of Molecular Microbiology and Immunology Insectary and Parasite Core facility, and Dr. Joel Tang from the Johns Hopkins NMR Core facility for his help with the NMR experiments. We would also like to thank the entire Casadevall Lab for their suggestions and inputs during conversations and their feedback during lab meetings and presentations. Figures in S9 Fig were made using BioRender.

\section{Author Contributions}

Conceptualization: Daniel F. Q. Smith, Emma Camacho, Raviraj Thakur, Alexander J. Barron, Nichole A. Broderick, Arturo Casadevall.

Data curation: Daniel F. Q. Smith, Alexander J. Barron.

Formal analysis: Daniel F. Q. Smith, Alexander J. Barron.

Funding acquisition: Emma Camacho, George Dimopoulos, Nichole A. Broderick, Arturo Casadevall.

Investigation: Daniel F. Q. Smith, Emma Camacho, Alexander J. Barron, Nichole A. Broderick.

Methodology: Daniel F. Q. Smith, Emma Camacho, Raviraj Thakur, Alexander J. Barron, Yuemei Dong, Nichole A. Broderick, Arturo Casadevall.

Project administration: Daniel F. Q. Smith, Emma Camacho, Alexander J. Barron, Nichole A. Broderick, Arturo Casadevall.

Resources: Emma Camacho, Raviraj Thakur, George Dimopoulos, Nichole A. Broderick, Arturo Casadevall.

Software: Alexander J. Barron.

Supervision: Emma Camacho, Raviraj Thakur, Nichole A. Broderick, Arturo Casadevall.

Validation: Daniel F. Q. Smith.

Visualization: Daniel F. Q. Smith, Alexander J. Barron.

Writing - original draft: Daniel F. Q. Smith.

Writing - review \& editing: Daniel F. Q. Smith, Emma Camacho, Raviraj Thakur, Alexander J. Barron, Yuemei Dong, George Dimopoulos, Nichole A. Broderick, Arturo Casadevall. 


\section{References}

1. Whitten MMA, Coates CJ. Re-evaluation of insect melanogenesis research: Views from the dark side. Pigment Cell Melanoma Res. 2017; 30(4):386-401. https://doi.org/10.1111/pcmr.12590 PMID: 28378380

2. Prota G. Recent Advances in the Chemistry of Melanogenesis in Mammals. J Investig Dermatol. 1980 Jul 1; 75(1):122-7. https://doi.org/10.1111/1523-1747.ep12521344 PMID: 6771336

3. Nappi AJ, Christensen BM. Melanogenesis and associated cytotoxic reactions: Applications to insect innate immunity. Insect Biochem Mol Biol. 2005 May 1; 35(5):443-59. https://doi.org/10.1016/j.ibmb. 2005.01.014 PMID: 15804578

4. Fujieda N, Murata M, Yabuta S, Ikeda T, Shimokawa C, Nakamura Y, et al. Multifunctions of MelB, a Fungal Tyrosinase from Aspergillus oryzae. Chembiochem. 2012; 13(2):193-201. https://doi.org/10. 1002/cbic.201100609 PMID: 22213164

5. Lerch K. Tyrosinase: Molecular and Active-Site Structure. In: Enzymatic Browning and Its Prevention [Internet]. Am Chem Soc. 1995 [cited 2020 Oct 21]. p. 64-80. (ACS Symposium Series; vol. 600). Available from: https://doi.org/10.1021/bk-1995-0600.ch005.

6. Galván I, Solano F. Bird Integumentary Melanins: Biosynthesis, Forms, Function and Evolution. Int J Mol Sci. 2016 Apr; 17(4):520. https://doi.org/10.3390/ijms17040520 PMID: 27070583

7. Martín-Durán JM, de A, Sebé-Pedrós A, Ruiz-Trillo I, Hejnol AA. Broad Genomic Survey Reveals Multiple Origins and Frequent Losses in the Evolution of Respiratory Hemerythrins and Hemocyanins. Genome Biol Evol. 2013 Jul 1; 5(7):1435-42. https://doi.org/10.1093/gbe/evt102 PMID: 23843190

8. Ramsden CA, Riley PA. Tyrosinase: The four oxidation states of the active site and their relevance to enzymatic activation, oxidation and inactivation. Bioorg Med Chem. 2014 Apr 15; 22(8):2388-95. https://doi.org/10.1016/j.bmc.2014.02.048 PMID: 24656803

9. Christensen BM, Li J, Chen C-C, Nappi AJ. Melanization immune responses in mosquito vectors. Trends Parasitol. 2005 Apr 1; 21(4):192-9. https://doi.org/10.1016/j.pt.2005.02.007 PMID: 15780842

10. González-Santoyo I, Córdoba-Aguilar A. Phenoloxidase: a key component of the insect immune system. Entomol Exp Appl. 2012; 142(1):1-16.

11. Marmaras VJ, Charalambidis ND, Zervas CG. Immune response in insects: the role of phenoloxidase in defense reactions in relation to melanization and sclerotization. Arch Insect Biochem Physiol. 1996; 31(2):119-33. https://doi.org/10.1002/(SICl)1520-6327(1996)31:2<119::AID-ARCH1>3.0.CO;2-V PMID: 8580494

12. Chen CC, Chen CS. Brugia bahangi: Effects of Melanization on the Uptake of Nutrients by Microfilariae in Vitro. Exp Parasitol. 1995 Aug 1; 81(1):72-8. https://doi.org/10.1006/expr.1995.1094 PMID: 7628569

13. Zhao $P$, Lu Z, Strand MR, Jiang H. Antiviral, anti-parasitic, and cytotoxic effects of 5,6-dihydroxyindole $(\mathrm{DHI})$, a reactive compound generated by phenoloxidase during insect immune response. Insect Biochem Mol Biol. 2011 Sep; 41(9):645-52. https://doi.org/10.1016/j.ibmb.2011.04.006 PMID: 21554953

14. Ezzati-Tabizi R, Talaei-Hassanloui R, Farrokhi N, Hossininaveh V, Alavi M. Haemolymph phenoloxidase activity of larval Plodia interpunctella and Galleria mellonella in response to Beauveria bassiana and Pseudomonas fluorescens. Int J Agric Innov Res. 2013; 2(2):217-20.

15. Vertyporokh L, Hułas-Stasiak M, Wojda I. Host-pathogen interaction after infection of Galleria mellonella with the filamentous fungus Beauveria bassiana. Insect Sci. 2020; 27(5):1079-89. https://doi. org/10.1111/1744-7917.12706 PMID: 31245909

16. Nayar JK University of F, Knight JW. Wounding increases intracellular encapsulation (melanization) of developing Brugia malayi (Nematoda: Filarioidea) larvae in thoracic muscles of Anopheles quadrimaculatus. Comp Biochem Phys A, Physiol (USA) [Internet]. 1995 [cited 2020 Oct 21]; Available from: https://agris.fao.org/agris-search/search.do? recordID=US9619494.

17. Nayar JK, Knight JW, Vickery AC. Intracellular Melanization in the Mosquito Anopheles quadrimaculatus (Diptera: Culicidae) Against the Filarial Nematodes, Brugia spp. (Nematoda: Filarioidea). J Med Entomol. 1989 May 1; 26(3):159-66. https://doi.org/10.1093/jmedent/26.3.159 PMID: 2724313

18. Yassine $\mathrm{H}$, Kamareddine L, Osta MA. The Mosquito Melanization Response Is Implicated in Defense against the Entomopathogenic Fungus Beauveria bassiana. PLoS Pathog. 2012 Nov 15; 8(11): e1003029. https://doi.org/10.1371/journal.ppat.1003029 PMID: 23166497

19. Zdybicka-Barabas A, Cytryńska M. Phenoloxidase activity in hemolymph of Galleria mellonella larvae challenged with Aspergillus oryzae. 2011 [cited 2020 Oct 21]; Available from: https://pubag.nal.usda. gov/catalog/5208902.

20. Dimopoulos G, Müller H-M, Levashina EA, Kafatos FC. Innate immune defense against malaria infection in the mosquito. Curr Opin Immunol. 2001 Feb 1; 13(1):79-88. https://doi.org/10.1016/s09527915(00)00186-2 PMID: 11154922 
21. Schnitger AKD, Kafatos FC, Osta MA. The Melanization Reaction Is Not Required for Survival of Anopheles gambiae Mosquitoes after Bacterial Infections. J Biol Chem. 2007 Jul 27; 282(30):218848. https://doi.org/10.1074/jbc.M701635200 PMID: 17537726

22. Dubovskiy IM, Kryukova NA, Glupov VV, Ratcliffe NA. Encapsulation and nodulation in insects. Invertebr Surviv J. 2016 Jul 7; 13(1):229-46.

23. Du M-H, Yan Z-W, Hao Y-J, Yan Z-T, Si F-L, Chen B, et al. Suppression of Laccase 2 severely impairs cuticle tanning and pathogen resistance during the pupal metamorphosis of Anopheles sinensis (Diptera: Culicidae). Parasit Vectors. 2017 Apr 4; 10(1):171. https://doi.org/10.1186/s13071-017-2118-4 PMID: 28376914

24. Qiao L, Du M, Liang X, Hao Y, He X, Si F, et al. Tyrosine Hydroxylase is crucial for maintaining pupal tanning and immunity in Anopheles sinensis. Sci Rep. 2016 Jul 15; 6(1):29835. https://doi.org/10. 1038/srep29835 PMID: 27416870

25. Goulson D. The insect apocalypse, and why it matters. Curr Biol. 2019 Oct 7; 29(19):R967-71. https:// doi.org/10.1016/j.cub.2019.06.069 PMID: 31593678

26. Nosanchuk JD, Ovalle R, Casadevall A. Glyphosate Inhibits Melanization of Cryptococcus neoformans and Prolongs Survival of Mice after Systemic Infection. J Infect Dis. 2001 Apr 1; 183(7):1093-9. https://doi.org/10.1086/319272 PMID: 11237835

27. Samsel A, Seneff S. Glyphosate pathways to modern diseases V: Amino acid analogue of glycine in diverse proteins. J Biol Phys Chem. 2016 Mar 30; 16(1):9-46.

28. Duke SO, Powles SB. Glyphosate: a once-in-a-century herbicide. Pest Manag Sci. 2008; 64(4):31925. https://doi.org/10.1002/ps.1518 PMID: 18273882

29. Dill GM. Glyphosate-resistant crops: history, status and future. Pest Manag Sci. 2008; 61(3):219-24.

30. Gianessi JC\& L. Herbicide Tolerant Soybeans: Why Growers Are Adopting Roundup Ready Varieties [Internet]. 1999 [cited 2018 Jun 20]. Available from: http://agbioforum.org/v2n2/v2n2a02-carpenter. htm.

31. Benbrook CM. Impacts of genetically engineered crops on pesticide use in the U.S. - the first sixteen years. Environ Sci Eur. 2012 Dec 1; 24(1):24.

32. Benbrook CM. Trends in glyphosate herbicide use in the United States and globally. Environ Sci Eur [Internet]. 2016 [cited 2018 Jun 20]; 28(1). Available from: https://www.ncbi.nlm.nih.gov/pmc/articles/ PMC5044953/. https://doi.org/10.1186/s12302-016-0070-0 PMID: 27752438

33. Bott S, Tesfamariam T, Candan H, Cakmak I, Römheld V, Neumann G. Glyphosate-induced impairment of plant growth and micronutrient status in glyphosate-resistant soybean (Glycine max L.). Plant Soil. 2008 Sep 11; 312(1):185.

34. Laitinen $P$, Rämö $S$, Siimes K. Glyphosate translocation from plants to soil-does this constitute a significant proportion of residues in soil? Plant Soil. 2007 Nov 1; 300(1):51-60.

35. Singh S, Kumar V, Datta S, Wani AB, Dhanjal DS, Romero R, et al. Glyphosate uptake, translocation, resistance emergence in crops, analytical monitoring, toxicity and degradation: a review. Environ Chem Lett. 2020 May 1; 18(3):663-702.

36. Laitinen $P$, Siimes K, Rämö S, Jauhiainen L, Eronen L, Oinonen S, et al. Effects of Soil Phosphorus Status on Environmental Risk Assessment of Glyphosate and Glufosinate-Ammonium. J Environ Qual. 2008; 37(3):830-8. https://doi.org/10.2134/jeq2007.0256 PMID: 18453404

37. Laitinen P, Rämö S, Nikunen U, Jauhiainen L, Siimes K, Turtola E. Glyphosate and phosphorus leaching and residues in boreal sandy soil. Plant Soil. 2009 Oct 1; 323(1):267-83.

38. Lupi L, Bedmar F, Puricelli M, Marino D, Aparicio VC, Wunderlin D, et al. Glyphosate runoff and its occurrence in rainwater and subsurface soil in the nearby area of agricultural fields in Argentina. Chemosphere. 2019 Jun 1; 225:906-14.

39. Edwards WM, Triplett GB, Kramer RM. A Watershed Study of Glyphosate Transport in Runoff 1. J Environ Qual. 1980 12/01; 9(4):661-5.

40. Silva V, Montanarella L, Jones A, Fernández-Ugalde O, Mol HGJ, Ritsema CJ, et al. Distribution of glyphosate and aminomethylphosphonic acid (AMPA) in agricultural topsoils of the European Union. Sci Total Environ. 2018 Apr 15; 621:1352-9. https://doi.org/10.1016/j.scitotenv.2017.10.093 PMID: 29042088

41. Battaglin WA, Meyer MT, Kuivila KM, Dietze JE. Glyphosate and Its Degradation Product AMPA Occur Frequently and Widely in U.S. Soils, Surface Water, Groundwater, and Precipitation. J Am Water Resour Assoc. 2014; 50(2):275-90.

42. Bento CPM, Yang X, Gort G, Xue S, van Dam R, Zomer P, et al. Persistence of glyphosate and aminomethylphosphonic acid in loess soil under different combinations of temperature, soil moisture and light/darkness. Sci Total Environ. 2016 Dec 1; 572:301-11. https://doi.org/10.1016/j.scitotenv.2016. 07.215 PMID: 27505263 
43. Yang X, Wang F, Bento CPM, Meng L, van R, Mol H, et al. Decay characteristics and erosion-related transport of glyphosate in Chinese loess soil under field conditions. Sci Total Environ. 2015 Oct 15;530-531:87-95. https://doi.org/10.1016/j.scitotenv.2015.05.082 PMID: 26026412

44. Laitinen P, Siimes K, Eronen L, Rämö S, Welling L, Oinonen S, et al. Fate of the herbicides glyphosate, glufosinate-ammonium, phenmedipham, ethofumesate and metamitron in two Finnish arable soils. Pest Manag Sci. 2006; 62(6):473-91. https://doi.org/10.1002/ps.1186 PMID: 16628542

45. Sviridov AV. Microbial degradation of glyphosate herbicides (Review). Appl Biochem Microbiol. 2015 Mar; 51(2):188-95. https://doi.org/10.7868/s0555109915020221 PMID: 26027353

46. Gill JPK, Sethi N, Mohan A, Datta S, Girdhar M. Glyphosate toxicity for animals. Environ Chem Lett. 2018 Jun $1 ; 16(2): 401-26$.

47. Druille M, Cabello MN, Omacini M, Golluscio RA. Glyphosate reduces spore viability and root colonization of arbuscular mycorrhizal fungi. Appl Soil Ecol. 2013 Feb 1; 64:99-103.

48. Schafer JR, Hallett SG, Johnson WG. Rhizosphere Microbial Community Dynamics in GlyphosateTreated Susceptible and Resistant Biotypes of Giant Ragweed (Ambrosia trifida). wees. 2014 Apr; 62 (2):370-81.

49. Zobiole LHS, Kremer RJ, Oliveira RS, Constantin J. Glyphosate affects micro-organisms in rhizospheres of glyphosate-resistant soybeans. J Appl Microbiol. 2011; 110(1):118-27. https://doi.org/10. 1111/j.1365-2672.2010.04864.x PMID: 20880215

50. Van AHC, He MM, Shin K, Mai V, Jeong KC, Finckh MR, et al. Environmental and health effects of the herbicide glyphosate. Sci Total Environ. 2018 Mar 1;616-617:255-68.

51. Ratcliff AW, Busse MD, Shestak CJ. Changes in microbial community structure following herbicide (glyphosate) additions to forest soils. Appl Soil Ecol. 2006 Dec 1; 34(2):114-24.

52. Busse MD, Ratcliff AW, Shestak CJ, Powers RF. Glyphosate toxicity and the effects of long-term vegetation control on soil microbial communities. Soil Biol Biochem. 2001 Oct 1; 33(12):1777-89.

53. Weaver MA, Krutz LJ, Zablotowicz RM, Reddy KN. Effects of glyphosate on soil microbial communities and its mineralization in a Mississippi soil. Pest Manag Sci. 2007; 63(4):388-93. https://doi.org/10. 1002/ps.1351 PMID: 17348071

54. Pochron S, Simon L, Mirza A, Littleton A, Sahebzada F, Yudell M. Glyphosate but not Roundup $®$ harms earthworms (Eisenia fetida). Chemosphere. 2020 Feb 1; 241:125017. https://doi.org/10.1016/j. chemosphere.2019.125017 PMID: 31605995

55. Krams IA, Kecko S, Jõers $P$, Trakimas $G$, Elferts D, Krams R, et al. Microbiome symbionts and diet diversity incur costs on the immune system of insect larvae. J Exp Biol. 2017 Nov 15; 220(22):420412. https://doi.org/10.1242/jeb.169227 PMID: 28939559

56. Lewis Z, Lizé A. Insect behaviour and the microbiome. Curr Opin Insect Sci. 2015 Jun 1; 9:86-90. https://doi.org/10.1016/j.cois.2015.03.003 PMID: 32846714

57. Weiss B, Aksoy S. Microbiome influences on insect host vector competence. Trends Parasitol. 2011 Nov 1; 27(11):514-22. https://doi.org/10.1016/j.pt.2011.05.001 PMID: 21697014

58. Lesperance DN, Broderick NA. Microbiomes as modulators of Drosophila melanogaster homeostasis and disease. Curr Opin Insect Sci. 2020 Jun 1; 39:84-90. https://doi.org/10.1016/j.cois.2020.03.003 PMID: 32339931

59. Motta EVS, Raymann K, Moran NA. Glyphosate perturbs the gut microbiota of honey bees. Proc Natl Acad Sci U S A. 2018 09; 115(41):10305-10. https://doi.org/10.1073/pnas.1803880115 PMID: 30249635

60. Blot N, Veillat L, Rouzé R, Delatte H. Glyphosate, but not its metabolite AMPA, alters the honeybee gut microbiota. PLoS ONE. 2019 Apr 16; 14(4):e0215466. https://doi.org/10.1371/journal.pone. 0215466 PMID: 30990837

61. Rio RVM, Jozwick AKS, Savage AF, Sabet A, Vigneron A, Wu Y, et al. Mutualist-Provisioned Resources Impact Vector Competency. MBio. 2019 Jun 25; 10(3):e00018-9. https://doi.org/10.1128/ mBio.00018-19 PMID: 31164458

62. Haughton AJ, Bell JR, Wilcox A, Boatman ND. The effect of the herbicide glyphosate on non-target spiders: Part I. Direct effects on Lepthyphantes tenuis under laboratory conditions. Pest Manag Sci. 2001; 57(11):1033-6. https://doi.org/10.1002/ps.388 PMID: 11721520

63. Evans SC, Shaw EM, Rypstra AL. Exposure to a glyphosate-based herbicide affects agrobiont predatory arthropod behaviour and long-term survival. Ecotoxicology. 2010 Oct 1; 19(7):1249-57. https:// doi.org/10.1007/s10646-010-0509-9 PMID: 20552395

64. Tahir HM, Basheer T, Ali S, Yaqoob R, Naseem S, Khan SY. Effect of Pesticides on Biological Control Potential of Neoscona theisi (Araneae: Araneidae). J Insect Sci [Internet]. 2019 Mar 1 [cited 2020 Oct 18]; 19(2). Available from: https://academic.oup.com/jinsectscience/article/19/2/17/5420491. https:// doi.org/10.1093/jisesa/iez024 PMID: 30915446 
65. Baglan H, Lazzari CR, Guerrieri FJ. Glyphosate impairs learning in Aedes aegypti mosquito larvae at field-realistic doses. J Exp Biol [Internet]. 2018 Oct 15 [cited 2020 Oct 14]; 221(20). Available from: https://jeb.biologists.org/content/221/20/jeb187518. https://doi.org/10.1242/jeb.187518 PMID: 30127074

66. Farina WM, Balbuena MS, Herbert LT, Mengoni Goñalons C, Vázquez DE. Effects of the Herbicide Glyphosate on Honey Bee Sensory and Cognitive Abilities: Individual Impairments with Implications for the Hive. Insects. 2019 Oct; 10(10):354. https://doi.org/10.3390/insects10100354 PMID: 31635293

67. Tomé HVV, Schmehl DR, Wedde AE, Godoy RSM, Ravaiano SV, Guedes RNC, et al. Frequently encountered pesticides can cause multiple disorders in developing worker honey bees. Environ Pollut. 2020 Jan 1; 256:113420. https://doi.org/10.1016/j.envpol.2019.113420 PMID: 31813703

68. Cuhra M, Traavik T, Dando M, Primicerio R, Holderbaum DF, Bøhn T. Glyphosate-Residues in Roundup-Ready Soybean Impair Daphnia magna Life-Cycle. J Agric Chem Environ. 2015 Jan 22; 4 (1):24-36.

69. Güngördü A. Comparative toxicity of methidathion and glyphosate on early life stages of three amphibian species: Pelophylax ridibundus, Pseudepidalea viridis, and Xenopus laevis. Aquat Toxicol. 2013 Sep 15;140-141:220-8.

70. El-Shenawy NS. Oxidative stress responses of rats exposed to Roundup and its active ingredient glyphosate. Environ Toxicol Pharmacol. 2009 Nov 1; 28(3):379-85. https://doi.org/10.1016/j.etap. 2009.06.001 PMID: 21784030

71. Uren Webster TM, Santos EM. Global transcriptomic profiling demonstrates induction of oxidative stress and of compensatory cellular stress responses in brown trout exposed to glyphosate and Roundup. BMC Genomics. 2015 Jan 31; 16(1):32. https://doi.org/10.1186/s12864-015-1254-5 PMID: 25636363

72. Nwani CD, Nagpure NS, Kumar R, Kushwaha B. Lakra WS. DNA damage and oxidative stress modulatory effects of glyphosate-based herbicide in freshwater fish, Channa punctatus. Environ Toxicol Pharmacol. 2013 Sep 1; 36(2):539-47. https://doi.org/10.1016/j.etap.2013.06.001 PMID: 23816461

73. de Aguiar LM, Figueira FH, Gottschalk MS, da CE. Glyphosate-based herbicide exposure causes antioxidant defence responses in the fruit fly Drosophila melanogaster. Comp Biochem Physiol C Toxicol Pharmacol. 2016 Jul 1;185-186:94-101. https://doi.org/10.1016/j.cbpc.2016.03.006 PMID: 26980113

74. Puértolas L, Damásio J, Barata C, Soares AMVM, Prat N. Evaluation of side-effects of glyphosate mediated control of giant reed(Arundo donax) on the structure and function of a nearby Mediterranean river ecosystem. Environ Res. 2010 Aug 1; 110(6):556-64. https://doi.org/10.1016/j.envres.2010.05. 004 PMID: 20541186

75. Kwiatkowska M, Nowacka-Krukowska H, Bukowska B. The effect of glyphosate, its metabolites and impurities on erythrocyte acetylcholinesterase activity. Environ Toxicol Pharmacol. 2014 May 1; 37 (3):1101-8. https://doi.org/10.1016/j.etap.2014.04.008 PMID: 24780534

76. Kwiatkowska M, Huras B, Bukowska B. The effect of metabolites and impurities of glyphosate on human erythrocytes (in vitro). Pestic Biochem Physiol. 2014 Feb 1; 109:34-43. https://doi.org/10. 1016/j.pestbp.2014.01.003 PMID: 24581382

77. Thompson PG, Smouse PE, Scofield DG, Sork VL. What seeds tell us about birds: a multi-year analysis of acorn woodpecker foraging movements. Mov Ecol. 2014 Jun 23; 2(1):12.

78. Mylonakis E, Moreno R, Khoury JBE, Idnurm A, Heitman J, Calderwood SB, et al. Galleria mellonella as a Model System To Study Cryptococcus neoformans Pathogenesis. Infect Immun. 2005 Jul 1; 73 (7):3842-50. https://doi.org/10.1128/IAI.73.7.3842-3850.2005 PMID: 15972469

79. Therneau TM. coxme: Mixed Effects Cox Models [Internet]. 2020 [cited 2021 Feb 7]. Available from: https://CRAN.R-project.org/package=coxme.

80. Smith RC, Vega-Rodríguez J, Jacobs-Lorena M, Smith RC, Vega-Rodríguez J, Jacobs-Lorena M. The Plasmodium bottleneck: malaria parasite losses in the mosquito vector. Mem Inst Oswaldo Cruz. 2014 Aug; 109(5):644-61. https://doi.org/10.1590/0074-0276130597 PMID: 25185005

81. Sinden RE, Billingsley PF. Plasmodium invasion of mosquito cells: hawk or dove? Trends Parasitol. 2001 May; 17(5):209-12. https://doi.org/10.1016/s1471-4922(01)01928-6 PMID: 11323288

82. Whitten MMA, Shiao SH, Levashina EA. Mosquito midguts and malaria: cell biology, compartmentalization and immunology. Parasite Immunol. 2006; 28(4):121-30. https://doi.org/10.1111/j.1365-3024. 2006.00804.x PMID: 16542314

83. Gouagna LC, Gouagna LC, Mulder B, Mulder B, Noubissi E, Noubissi E, et al. The early sporogonic cycle of Plasmodium falciparum in laboratory-infected Anopheles gambiae: an estimation of parasite efficacy. Tropical Med Int Health. 1998; 3(1):21-8. https://doi.org/10.1046/j.1365-3156.1998.00156.x PMID: 9484964 
84. Dong Y, Manfredini F, Dimopoulos G. Implication of the Mosquito Midgut Microbiota in the Defense against Malaria Parasites. PLoS Pathog [Internet]. 2009 May 8 [cited 2020 Apr 4]; 5(5). Available from: https://www.ncbi.nlm.nih.gov/pmc/articles/PMC2673032/. https://doi.org/10.1371/journal.ppat. 1000423 PMID: 19424427

85. Bahia AC, Dong Y, Blumberg BJ, Mlambo G, Tripathi A, BenMarzouk-Hidalgo OJ, et al. Exploring Anopheles gut bacteria for Plasmodium blocking activity. Environ Microbiol. 2014 Sep; 16(9):2980-94. https://doi.org/10.1111/1462-2920.12381 PMID: 24428613

86. Bai L, Wang L, Vega-Rodríguez J, Wang G, Wang SA. Gut Symbiotic Bacterium Serratia marcescens Renders Mosquito Resistance to Plasmodium Infection Through Activation of Mosquito Immune Responses. Front Microbiol. 2019; 10:1580. https://doi.org/10.3389/fmicb.2019.01580 PMID: 31379768

87. Romoli $\mathrm{O}$, Gendrin $\mathrm{M}$. The tripartite interactions between the mosquito, its microbiota and Plasmodium. Parasit Vectors. 2018 20; 11(1):200. https://doi.org/10.1186/s13071-018-2784-x PMID: 29558973

88. Mason HS. The chemistry of melanin; mechanism of the oxidation of dihydroxyphenylalanine by tyrosinase. J Biol Chem. 1948 Jan; 172(1):83-99. PMID: 18920770

89. Raper HS. The Tyrosinase-tyrosine Reaction. Biochem J. 1927; 21(1):89-96. https://doi.org/10.1042/ bj0210089 PMID: 16743827

90. García-Borrón JC, Sánchez MCO. Biosynthesis of Melanins. In: Melanins and Melanosomes [Internet]. John Wiley \& Sons, Ltd; 2011 [cited 2019 Jun 8]. p. 87-116. Available from: https://onlinelibrary. wiley.com/doi/abs/10.1002/9783527636150.ch4.

91. Jara JR, Solano F, Lozano JA. Assays for Mammalian Tyrosinase: A Comparative Study. Pigment Cell Res. 1988; 1(5):332-9. https://doi.org/10.1111/j.1600-0749.1988.tb00128.x PMID: 2907130

92. Chen Q-X, Song K-K, Wang Q, Huang H. Inhibitory Effects on Mushroom Tyrosinase by Some Alkylbenzaldehydes. J Enzyme Inhib Med Chem. 2003 Dec 1; 18(6):491-6. https://doi.org/10.1080/ 14756360310001613094 PMID: 15008513

93. Liu H-J, Ji S, Fan Y-Q, Yan L, Yang J-M, Zhou H-M, et al. The Effect of D-(-)-arabinose on Tyrosinase: An Integrated Study Using Computational Simulation and Inhibition Kinetics [Internet]. Vol. 2012, Enzyme Res. Hindawi; 2012 [cited 2020 Oct 27]. p. e731427. Available from: https://www.hindawi. com/journals/er/2012/731427/. https://doi.org/10.1155/2012/731427 PMID: 23365724

94. Glass RL. Metal complex formation by glyphosate. J Agric Food Chem. 1984 Nov 1; 32(6):1249-53.

95. Madsen HEL, Christensen HH, Gottlieb-Petersen C, Andresen AF, Smidsrød O, Pontchour C-O, et al. Stability Constants of Copper(II), Zinc, Manganese(II), Calcium, and Magnesium Complexes of N(Phosphonomethyl)glycine (Glyphosate). Acta Chem Scand. 1978;32a:79-83.

96. Zhou C-F, Wang Y-J, Li C-C, Sun R-J, Yu Y-C, Zhou D-M. Subacute toxicity of copper and glyphosate and their interaction to earthworm (Eisenia fetida). Environ Pollut. 2013 Sep 1; 180:71-7. https://doi. org/10.1016/j.envpol.2013.05.016 PMID: 23733011

97. Riley PA. Radicals in Melanin Biochemistry a. Ann N Y Acad Sci. 1988; 551(1):111-9.

98. Gordon MH. The Mechanism of Antioxidant Action in Vitro. In: Hudson BJF, editor. Food Antioxidants [Internet]. Dordrecht: Springer Netherlands; 1990 [cited 2019 Jun 8]. p. 1-18. (Elsevier Applied Food Science Series). Available from: https://doi.org/10.1007/978-94-009-0753-9_1.

99. Choe E, Min DB. Mechanisms of Antioxidants in the Oxidation of Foods. Compr Rev Food Sci Food Saf. 2009; 8(4):345-58.

100. Jodko-Piórecka K, Litwinienko G. Antioxidant activity of dopamine and L-DOPA in lipid micelles and their cooperation with an analogue of $\alpha$-tocopherol. Free Radic Biol Med. 2015 Jun 1; 83:1-11. https:// doi.org/10.1016/j.freeradbiomed.2015.02.006 PMID: 25701434

101. Bailey SI, Ritchie IM. A cyclic voltammetric study of the aqueous electrochemistry of some quinones. Electrochim Acta. 1985 Jan 1; 30(1):3-12.

102. Kissinger PT, Heineman WR. Cyclic voltammetry. J Chem Educ. 1983 Sep 1; 60(9):702.

103. Liu X, Zhang Z, Cheng G, Dong S. Spectroelectrochemical and Voltammetric Studies of L-DOPA. Electroanalysis. 2003; 15(2):103-7.

104. Fotouhi L, Tammari E, Asadi S, Heravi MM, Nematollahi D. Estimation of heterogeneous rate constants of reaction of electrochemically generated o-benzoquinones with various nucleophiles containing thiol group. Int J Chem Kinet. 2009 Jun 1; 41(6):426-31.

105. Boyle JH, Dalgleish HJ, Puzey JR. Monarch butterfly and milkweed declines substantially predate the use of genetically modified crops. Proc Natl Acad Sci U S A. 2019 Feb 19; 116(8):3006-11. https://doi. org/10.1073/pnas.1811437116 PMID: 30723147 
106. Wepprich T, Adrion JR, Ries L, Wiedmann J, Haddad NM. Butterfly abundance declines over 20 years of systematic monitoring in Ohio, USA. PLoS ONE. 2019 Jul 9; 14(7):e0216270. https://doi.org/10. 1371/journal.pone.0216270 PMID: 31287815

107. Wepprich T. Monarch butterfly trends are sensitive to unexamined changes in museum collections over time. PNAS. 2019 Jul 9; 116(28):13742-4. https://doi.org/10.1073/pnas.1903511116 PMID: 31291703

108. Thogmartin WE, Wiederholt R, Oberhauser K, Drum RG, Diffendorfer JE, Altizer S, et al. Monarch butterfly population decline in North America: identifying the threatening processes. R Soc Open Sci; 4 (9):170760. https://doi.org/10.1098/rsos.170760 PMID: 28989778

109. Molina-Cruz A, DeJong RJ, Ortega C, Haile A, Abban E, Rodrigues J, et al. Some strains of Plasmodium falciparum, a human malaria parasite, evade the complement-like system of Anopheles gambiae mosquitoes. PNAS. 2012 Jul 10; 109(28):E1957-62. https://doi.org/10.1073/pnas.1121183109 PMID: 22623529

110. Urabe K, Aroca P, Tsukamoto K, Mascagna D, Palumbo A, Prota G, et al. The inherent cytotoxicity of melanin precursors: A revision. Biochimica et Biophysica Acta (BBA)—Molecular Cell Research. 1994 Apr 28; 1221(3):272-8. https://doi.org/10.1016/0167-4889(94)90250-x PMID: 8167148

111. Smith RC, Plasmodium Oocysts B-MC. Overlooked Targets of Mosquito Immunity. Trends Parasitol. 2016; 32(12):979-90. https://doi.org/10.1016/j.pt.2016.08.012 PMID: 27639778

112. Hogg JC, Hurd $\mathrm{H}$. The effects of natural Plasmodium falciparum infection on the fecundity and mortality of Anopheles gambiae s. I in north east Tanzania. Parasitology. 1997 Apr; 114(Pt 4):325-31. https://doi.org/10.1017/s0031182096008542 PMID: 9107019

113. Okech BA, Gouagna LC, Kabiru EW, Beier JC, Yan G, Githure JI. Influence of age and previous diet of Anopheles gambiae on the infectivity of natural Plasmodium falciparum gametocytes from human volunteers. J Insect Sci [Internet]. 2004 Oct 22 [cited 2020 Oct 26];4. Available from: https://www.ncbi. nlm.nih.gov/pmc/articles/PMC1081565/. https://doi.org/10.1093/jis/4.1.33 PMID: 15861248

114. Bando H, Okado K, Guelbeogo WM, Badolo A, Aonuma H, Nelson B, et al. Intra-specific diversity of Serratia marcescens in Anopheles mosquito midgut defines Plasmodium transmission capacity. Sci Rep. 2013 Apr 10; 3(1):1-9. https://doi.org/10.1038/srep01641 PMID: 23571408

115. Cirimotich CM, Dong Y, Clayton AM, Sandiford SL, Souza-Neto JA, Mulenga M, et al. Natural Microbe-Mediated Refractoriness to Plasmodium Infection in Anopheles gambiae. Science. 2011 May 13; 332(6031):855-8. https://doi.org/10.1126/science.1201618 PMID: 21566196

116. Zhao H, Li G, Guo D, Wang Y, Liu Q, Gao Z, et al. Transcriptomic and metabolomic landscape of the molecular effects of glyphosate commercial formulation on Apis mellifera ligustica and Apis cerana cerana. Sci Total Environ. 2020 Nov 20; 744:140819. https://doi.org/10.1016/j.scitotenv.2020.140819 PMID: 32693280

117. Benoit JB, Vigneron A, Broderick NA, Wu Y, Sun JS, Carlson JR, et al. Symbiont-induced odorant binding proteins mediate insect host hematopoiesis. eLife [Internet]. 2017 [cited 2021 Feb 7]; 6. Available from: https://www.ncbi.nlm.nih.gov/pmc/articles/PMC5231409/. https://doi.org/10.7554/eLife. 19535 PMID: 28079523

118. Dzhavakhiya V, Voinova TM, Popletaeva S, Statsyuk N, Mikityuk O, Nazarova TA, et al. Some natural and synthetic compounds inhibiting the biosynthesis of aflatoxin B1 and melanin in Aspergillus flavus. SEL'SKOKHOZYAISTVENNAYA BIOLOGIA. 2016 Sep 1; 51:533-42.

119. Yu RJ, Scott EJV. N- (phosphonoalkyl)-amino acids, derivatives thereof and compositions and methods of use [Internet]. US7429575B2, 2008 [cited 2018 Jun 19]. Available from: https://patents.google. $\mathrm{com} /$ patent/US7429575B2/en?q=melanin\&q=aminophosphonic+acid\&q=A61Q19\%2f02.

120. Seguin $M-C$, Babizhayev MA. Cosmetic composition useful notably for the skin whitening and melanogenesis inhibiting agent containing such a cosmetic composition [Internet]. US6280715B1, 2001 [cited 2018 Jun 19]. Available from: https://patents.google.com/patent/US6280715B1/en.

121. Jiang N, Sun N, Xiao D, Pan J, Wang Y, Zhu X. A copper-responsive factor gene CUF1 is required for copper induction of laccase in Cryptococcus neoformans. FEMS Microbiol Lett. 2009 Jul 1; 296(1):8490. https://doi.org/10.1111/j.1574-6968.2009.01619.x PMID: 19459959

122. Nenadis N, Wang L-F, Tsimidou M, Zhang H-Y. Estimation of Scavenging Activity of Phenolic Compounds Using the ABTS•+ Assay. J Agric Food Chem. 2004 Jul 1; 52(15):4669-74. https://doi.org/10. 1021/jf0400056 PMID: 15264898

123. Yu RJ, Scott EJV. Alpha hydroxyacids, alpha ketoacids and their use in treating skin conditions [Internet]. US4363815A, 1982 [cited 2019 Jun 8]. Available from: https://patents.google.com/patent/ US4363815A/en.

124. Brauman KA, Flörke M, Mueller ND, Foley JA. Widespread Occurrence of Glyphosate and its Degradation Product (AMPA) in U.S. Soils, Surface Water, Groundwater, and Precipitation, 2001-2009. AGU Fall Meeting Abstracts. 2011 Dec 1; 44:H44A-08. 
125. Hallmann CA, Sorg M, Jongejans E, Siepel H, Hofland N, Schwan H, et al. More than 75 percent decline over 27 years in total flying insect biomass in protected areas. PLoS One. 2017; 12(10): e0185809. https://doi.org/10.1371/journal.pone.0185809 PMID: 29045418

126. Jarvis B. The Insect Apocalypse Is Here. The New York Times [Internet]. 2018 Nov 27 [cited 2020 May 6]; Available from: https://www.nytimes.com/2018/11/27/magazine/insect-apocalypse.html.

127. Montgomery GA, Dunn RR, Fox R, Jongejans E, Leather SR, Saunders ME, et al. Is the insect apocalypse upon us? How to find out. Biol Conserv. 2020 Jan 1; 241:108327.

128. Cardoso $P$, Leather SR. Predicting a global insect apocalypse. Insect Conserv Divers. 2019; 12 (4):263-7.

129. Saunders ME, Janes JK, O'Hanlon JC. Moving On from the Insect Apocalypse Narrative: Engaging with Evidence-Based Insect Conservation. Bioscience. 2020 Jan 1; 70(1):80-9.

130. Rosenheim JA, Ward PS. Insect diversity over 36 years at a protected Sierra Nevada (California) site: towards an evaluation of the insect apocalypse hypothesis. Ecol Entomol [Internet]. [cited 2020 Oct 25];n/a(n/a). Available from: https://onlinelibrary. wiley.com/doi/abs/10.1111/een.12888.

131. Saunders ME. Ups and downs of insect populations. Nat Ecol Evol. 2019 Dec; 3(12):1616-7. https:// doi.org/10.1038/s41559-019-1038-4 PMID: 31712695

132. Cornet S, Gandon S, Rivero A. Patterns of phenoloxidase activity in insecticide resistant and susceptible mosquitoes differ between laboratory-selected and wild-caught individuals. Parasit Vectors. 2013 Oct 31; 6(1):315. https://doi.org/10.1186/1756-3305-6-315 PMID: 24499651

133. Rodrigues J, Brayner FA, Alves LC, Dixit R, Barillas-Mury C. Hemocyte Differentiation Mediates Innate Immune Memory in Anopheles gambiae Mosquitoes. Science. 2010 Sep 10; 329(5997):1353-5. https://doi.org/10.1126/science.1190689 PMID: 20829487

134. Schindelin J, Arganda-Carreras I, Frise E, Kaynig V, Longair M, Pietzsch T, et al. Fiji: an open-source platform for biological-image analysis. Nat Methods. 2012 Jul; 9(7):676-82. https://doi.org/10.1038/ nmeth.2019 PMID: 22743772

135. Schloss PD, Westcott SL, Ryabin T, Hall JR, Hartmann M, Hollister EB, et al. Introducing mothur: Open-Source, Platform-Independent, Community-Supported Software for Describing and Comparing Microbial Communities. Appl Environ Microbiol. 2009 Dec 1; 75(23):7537-41. https://doi.org/10.1128/ AEM.01541-09 PMID: 19801464

136. Chong J, Liu P, Zhou G, Xia J. Using MicrobiomeAnalyst for comprehensive statistical, functional, and meta-analysis of microbiome data. Nat Protoc. 2020 Mar; 15(3):799-821. https://doi.org/10.1038/ s41596-019-0264-1 PMID: 31942082

137. Dhariwal A, Chong J, Habib S, King IL, Agellon LB, Xia J. MicrobiomeAnalyst: a web-based tool for comprehensive statistical, visual and meta-analysis of microbiome data. Nucleic Acids Res. 201703 ; 45(W1):W180-8. https://doi.org/10.1093/nar/gkx295 PMID: 28449106

138. Winder AJ, Harris $\mathrm{H}$. New assays for the tyrosine hydroxylase and dopa oxidase activities of tyrosinase. Eur J Biochem. 1991 Jun 1; 198(2):317-26. https://doi.org/10.1111/j.1432-1033.1991.tb16018. x PMID: 1674912

139. Maurya DK, Devasagayam TPA. Antioxidant and prooxidant nature of hydroxycinnamic acid derivatives ferulic and caffeic acids. Food Chem Toxicol. 2010 Dec; 48(12):3369-73. https://doi.org/10. 1016/j.fct.2010.09.006 PMID: 20837085 